\title{
Physical states in the canonical tensor model from the perspective of random tensor networks
}

\author{
Gaurav Narain, ${ }^{a}$ Naoki Sasakura ${ }^{b}$ and Yuki Sato ${ }^{c}$ \\ ${ }^{a}$ The Institute for Fundamental Study "The Tah Poe Academia Institute", \\ Naresuan University, Phitsanulok 65000, Thailand \\ ${ }^{b}$ Yukawa Institute for Theoretical Physics, \\ Kyoto University, Kyoto 606-8502, Japan \\ ${ }^{c}$ National Institute for Theoretical Physics, \\ School of Physics and Centre for Theoretical Physics, \\ University of the Witwartersrand, WITS 2050, South Africa \\ E-mail: gaunarain@nu.ac.th, sasakura@yukawa.kyoto-u.ac.jp, \\ Yuki.Sato@wits.ac.za
}

ABSTRACT: Tensor models, generalization of matrix models, are studied aiming for quantum gravity in dimensions larger than two. Among them, the canonical tensor model is formulated as a totally constrained system with first-class constraints, the algebra of which resembles the Dirac algebra of general relativity. When quantized, the physical states are defined to be vanished by the quantized constraints. In explicit representations, the constraint equations are a set of partial differential equations for the physical wave-functions, which do not seem straightforward to be solved due to their non-linear character. In this paper, after providing some explicit solutions for $N=2,3$, we show that certain scale-free integration of partition functions of statistical systems on random networks (or random tensor networks more generally) provides a series of solutions for general $N$. Then, by generalizing this form, we also obtain various solutions for general $N$. Moreover, we show that the solutions for the cases with a cosmological constant can be obtained from those with no cosmological constant for increased $N$. This would imply the interesting possibility that a cosmological constant can always be absorbed into the dynamics and is not an input parameter in the canonical tensor model. We also observe the possibility of symmetry enhancement in $N=3$, and comment on an extension of Airy function related to the solutions.

KEYwords: Models of Quantum Gravity, Non-Commutative Geometry

ARXIV EPRINT: 1410.2683 


\section{Contents}

1 Introduction 1

2 Canonical tensor model $\quad 4$

$3 \quad N=2$ model $\quad 8$

3.1 Case without a cosmological constant 8

$\begin{array}{ll}3.2 & \text { Case with a cosmological constant } \\ \end{array}$

$4 \quad N=3$ model $\quad 13$

$\begin{array}{lll}4.1 & \text { Case with no cosmological constant } & 13\end{array}$

$\begin{array}{ll}4.2 \text { Case with a cosmological constant } & 16\end{array}$

5 Solutions in terms of statistical systems on random networks 18

6 Simpler solutions $\quad 22$

7 Explicit examples $\quad 24$

$\begin{array}{lll}7.1 & N=1 & 24\end{array}$

$\begin{array}{ll}7.2 N=2 & 24\end{array}$

8 Solutions in $P$-representation without a cosmological constant 25

9 Solutions with integration over matrix and tensor variables $\quad 27$

10 Solutions in $P$-representation with a cosmological constant 29

11 A theorem for ignoring a cosmological constant 32

$\begin{array}{ll}12 \text { Summary and discussions } & 34\end{array}$

\section{Introduction}

Tensor models were initially introduced as models for quantum gravity in $D>2$ dimensions [1-3], by generalizing the matrix models [4], which are known to consistently describe the $D=2$ dimensional quantum gravity. The main idea of the tensor models was that the Feynman diagrams obtained via perturbative treatment of the tensor models could be identified with the dual diagrams of simplicial manifolds for $D>2$ dimensions, the summation of which would evaluate in a continuum limit the geometric sum in Euclidean quantum gravity. However, these original tensor models were unsuccessful due to singular or un-oriented Feynman diagrams $[2,5]$, and also it was not clear how to take continuum 
limits, because of the absence of $1 / N$ expansions, ${ }^{1}$ which played essential roles in taking the continuum limits of the matrix models. This situation has been drastically changed by the advent of the colored tensor models [6]. The colored tensor models have a one-to-one correspondence between the Feynman diagrams and pseudo-simplicial manifolds, and also have $1 / N$ expansions, the leading terms of which are composed of melonic diagrams [7]. The melonic diagrams correspond to simplicial manifolds with spherical topology, but are geometrically singular [7-9]. Therefore it seems important to modify the leading order or to include higher order $1 / N$ corrections to obtain continuum limits of physical interests as in the matrix models. A number of efforts are currently being made in these directions [10-19]. The colored tensor models have also stimulated the developments of the renormalization group procedure of group field theories [20-30], which are tensor models with group-valued indices [31, 32] and are studied extensively in the context of the loop quantum gravity [33-35].

The developments above of tensor models concern the Euclidean case. In quantum gravity with geometric fluctuations, it would be unclear whether the time-like direction can be treated in the same way as the other space-like directions, which is the standard procedure with Wick rotation in field theories on flat space-times. In fact, in the dynamical triangulation model of quantum gravity, it has been shown that simplicial complexes which can be regarded as smooth space-times in global scale dominate in Causal Dynamical Triangulation [36], while Dynamical Triangulation, the original Euclidean model, does not seem successful in this respect. ${ }^{2}$ Inspired by this fact, one of the present authors proposed Hamilton formalism of tensor models, dubbed the canonical tensor model in short [39-41]. ${ }^{3}$ This model has a canonical conjugate pair of three-index tensors as dynamical variables, and is formulated as a totally constrained system with first-class constraints, the algebra of which resembles that of the $\mathrm{ADM}$ formalism [43, 44] of general relativity. A number of intriguing results have been obtained so far in this direction: there exists a formal continuum limit in which the first-class constraint algebra agrees with that of the ADM formalism [39], the model is unique under some physically reasonable assumptions [40], the constraints can consistently be quantized to form a first-class quantized constraint algebra with no anomaly [45], locality is favored at least for $N=2$ in the physical wave-function satisfying the quantized constraints [45], the $N=1$ case classically agrees with the minisuperspace approximation of general relativity [46], and the model with $N=2$ has intimate relations with Ising model on random networks [47, 48].

While the above results suggest that the canonical tensor model can be an interesting model of quantum gravity, a major question on the validity of the model would be whether its quantum dynamics can produce an object like space-time. This question may be answered by studying the physical states (or physical wave-functions in explicit representations) which satisfy the quantized first-class constraints of the canonical tensor

\footnotetext{
${ }^{1} N$ denotes the dimension of the vector space associated to the indices of vectors, matrices and tensors in this paper. Namely, an index runs through $1,2, \cdots, N$.

${ }^{2}$ When coupling many U(1)-fields, the authors in [37] found a promise of a phase transition higher than first order, which, however, is in conflict with the result in [38].

${ }^{3} \mathrm{An}$ operator formalism of group field theories has been developed in [42].
} 
model. ${ }^{4}$ This is rather a difficult task due to the non-linear character of the quantized constraints and so far has been solved only in the case of $N=2$ [45]. On the other hand, the previous paper [47] implies the intimate relations between the canonical tensor model and statistical systems on random networks [48, 49]. In fact, the present paper successfully constructs physical wave-functions satisfying the quantized constraints, general for arbitrary integer $N$, in terms of scale free integration of "grand-type" partition functions of statistical systems on random networks (or rather random tensor networks as we will see). We also obtain other physical wave-functions either by generalizing them for any $N$ or by explicitly solving the differential equations representing the constraints for $N=2,3$. The following two properties of the solutions suggest physically interesting directions of future study. One is that the wave-functions can have peaks at configurations where symmetries are enhanced. This is explicitly observed for $N=3$, and we expect this to be true also for higher $N$. In future study, this feature may provide a clue as to why our universe is isotropic and homogenous at large scales. We also find that the physical wave-functions satisfying the constraints for $N=m$ with a cosmological constant can be obtained by restricting the domains of those for $N=m+1$ with no cosmological constant. This suggests that, in the canonical tensor model, a cosmological constant is not an input parameter, but rather a part of dynamics. This would provide an interesting clue to the problem of the cosmological constant in future study.

This paper is organized as follows. In section 2, we recapitulate the canonical tensor model and its quantized constraints with a cosmological constant. A classification of physical states in terms of their kinematical and dynamical natures is introduced. In section 3, we obtain the physical wave-functions satisfying the quantized constraints for $N=2$ generally with a cosmological constant by explicitly writing down and solving the partial differential equations. In section 4 , we obtain physical wave-functions on a $Z_{3}$ symmetric subspace of the configuration space in the case of $N=3$ generally with a cosmological constant. We find that the wave-functions can have peaks at configurations with enhanced symmetries. In section 5, we construct physical wave-functions for general $N$ with no cosmological constant in terms of scale free integration of partition functions of statistical systems on random networks. In section 6 , we obtain physical wave-functions with simpler expressions by generalizing the result in section 5 . In section 7 , we explicitly evaluate the wave-functions in section 6 for $N=1,2$ to check the validity of the expressions. In section 8 and 9 , we work in a momentum representation and obtain other physical wave-functions for the case of no cosmological constant and general $N$. We also obtain other types of physical wave-functions in terms of integration over matrix and tensor variables. In section 10, we obtain physical wave-functions for general $N$ with a cosmological constant by generalizing the results in section 8 . We find that these wave-functions for $N=m$ can be obtained by restricting the domains of the wave-functions for $N=m+1$ with no cosmological constant. A general theorem of this aspect is given in section 11. Finally, section 12 is devoted to summary and discussions.

\footnotetext{
${ }^{4}$ In analogy with Hamilton formalism of general relativity, the quantized constraint equations and the physical wave-functions respectively correspond to the Wheeler-DeWitt equations and the Wheeler-DeWitt wave-functions [50].
} 


\section{Canonical tensor model}

The canonical tensor model has been introduced as a theory of dynamical fuzzy spaces [39$41,45]$, aiming to construct a quantum theory of gravity. First of all, the fuzzy space is an extended notion of space defined by a set of functions, $f_{a}(a=1,2, \cdots, N)$, and the product of such functions:

$$
f_{a} \star f_{b}=M_{a b}^{c} f_{c},
$$

where $M_{a b}{ }^{c}$ is a three-index tensor characterizing the fuzzy space. For instance, in the case of ordinary $d$-dimensional Euclidean space, (2.1) is given by

$$
f_{z_{1}} \star f_{z_{2}}=\delta^{d}\left(z_{1}-z_{2}\right) f_{z_{1}} .
$$

Here $f_{z}$ can be expressed by a $d$-dimensional delta function, $f_{z}=\delta^{d}(x-z)$ where $x, z \in \mathbb{R}^{d}$. This implies that points in Euclidean space correspond to functions, $f_{z}$ 's. As the threeindex tensor is a delta function, distant points (functions) are independent. One can extend this notion of localized points to non-local "fuzzy" ones using a non-trivial three-index tensor, $M_{a b}{ }^{c}$. Therefore, in general a fuzzy space is non-local and can be of any dimension. In the canonical tensor model, in order to define the physics controlling the dynamics, two external conditions are imposed [51]. These are the reality conditions given by

$$
f_{a}^{*}=f_{a}, \quad\left(f_{a} \star f_{b}\right)^{*}=f_{b} \star f_{a},
$$

where $*$ means complex conjugation, and the trace-like property of the inner product,

$$
\left\langle f_{a} \mid f_{b} \star f_{c}\right\rangle=\left\langle f_{a} \star f_{b} \mid f_{c}\right\rangle=\left\langle f_{c} \star f_{a} \mid f_{b}\right\rangle,
$$

where the inner product, $\left\langle f_{a} \mid f_{b}\right\rangle$, has been chosen to be real, symmetric and bilinear. If one initially sets the inner product to be positive-definite, one can choose an orthonormal basis:

$$
\left\langle f_{a} \mid f_{b}\right\rangle=\delta_{a b},
$$

by a real linear transformation preserving the two conditions stated in (2.3) and (2.4). This choice of orthonormal basis in (2.5) allows to rewrite (2.1) in the following form:

$$
M_{a b c}=\left\langle f_{a} \star f_{b} \mid f_{c}\right\rangle=M_{a b}{ }^{d}\left\langle f_{d} \mid f_{c}\right\rangle .
$$

Using the three-index tensor $M_{a b c}$ from (2.6), it is possible to extract the dynamics of the fuzzy space under the imposed conditions stated in (2.3) and (2.4). The inner product in (2.5) is $O(N)$ invariant, which appears in the transformations of $M$ as

$$
M_{a b c}^{\prime}=L_{a}{ }^{d} L_{b}{ }^{e} L_{c}{ }^{f} M_{d e f}, \quad L \in O(N) .
$$

This symmetry serves as a kinematical symmetry of the canonical tensor model. The two conditions, (2.3) and (2.4), lead to the generalized hermiticity condition of the threeindex tensor:

$$
M_{a b c}=M_{b c a}=M_{c a b}=M_{b a c}^{*}=M_{a c b}^{*}=M_{c b a}^{*} .
$$


The canonical tensor model stands on the position such that space-time would be a time evolution of the dynamical fuzzy space satisfying (2.3) and (2.4), or equivalently the generalized hermiticity condition, (2.8). This can be realized in analogy with the ADM formalism of general relativity $[43,44]$. In the ADM formalism of general relativity, one considers the 4-dimensional space-time as a time propagation (history) of the 3-dimensional spatial hyper-surface, or in other words, parametrizes the metric in terms of non-dynamical fields, $N, N^{i}(i=1,2,3)$, and dynamical spatial metric, $h_{i j}$ :

$$
d s^{2}=-N^{2} d t^{2}+h_{i j}\left(N^{i} d t+d x^{i}\right)\left(N^{j} d t+d x^{j}\right) .
$$

Then one converts the Einstein-Hilbert action into the Hamiltonian using the spatial metric, $h_{i j}$, and its conjugate momentum, $p^{i j}$, as phase-space variables. As a result of general covariance, the Hamiltonian can be written as a linear combination of constraints:

$$
H_{\mathrm{ADM}}=\int d^{3} x\left[N(x) \mathcal{H}(x)+N^{i}(x) \mathcal{H}_{i}(x)\right],
$$

where $\mathcal{H}$ and $\mathcal{H}_{i}$ are the generators of temporal and spatial diffeomorphism, respectively. They are constraints, classified as the first class following Dirac's theory of constrained systems. In fact, the constrains form a closed algebra. The canonical tensor model has been constructed in the same spirt of the ADM formalism, i.e., considering the threeindex tensor, $M_{a b c}$, and its conjugate momentum, $P_{a b c}$, as dynamical phase-space variables, with the model being introduced as a totally constrained Hamiltonian system. One can construct the generators corresponding to the $O(N)$-kinematical symmetry, (2.7), playing a similar role as the spatial diffeomorphism in general relativity, and remarkably one can uniquely determine the analogue of the temporal diffeomorphism of general relativity, under reasonable assumptions requiring closed constraint algebra, cubic terms at most, invariance under the time-reversal symmetry and connectivity [40].

In this paper we are interested in quantizing the canonical tensor model started in [45]. In the quantum version, one replaces the dynamical variable $M_{a b c}$ and its conjugate by corresponding operators $\hat{M}_{a b c}$ and its conjugate partner, respectively. This will be explained later in more detail. Besides, we especially consider the minimal version of the canonical tensor model dubbed minimal model [41], defined by a canonical conjugate pair of real and symmetric tensors, $\left(\hat{M}_{a b c}, \hat{P}_{a b c}\right)$,

$$
\hat{M}_{a b c}=\hat{M}_{a b c}^{\dagger}=\hat{M}_{b c a}=\hat{M}_{c a b}=\hat{M}_{b a c}=\hat{M}_{a c b}=\hat{M}_{b c a}, \text { and similarly for } \hat{P}_{a b c},
$$

which satisfy the following commutation relations:

$$
\left[\hat{M}_{a b c}, \hat{P}_{d e f}\right]=\frac{i}{6} \sum_{\sigma} \delta_{a \sigma(d)} \delta_{b \sigma(e)} \delta_{c \sigma(f)}, \quad\left[\hat{M}_{a b c}, \hat{M}_{d e f}\right]=\left[\hat{P}_{a b c}, \hat{P}_{d e f}\right]=0
$$

where the summation over $\sigma$ implies that over all the permutations of $d, e, f$. As the minimal model has less degrees of freedom, its quantization is easier to perform and the algebra is more tractable compared to the non-minimal version of the canonical tensor model. However the minimal model is still complicated enough to capture the non-trivial 
physics of the system. It therefore becomes a good theoretical laboratory to gain expertise and maturity in the subject (see, say [45]). The quantum dynamics of the minimal model can be described through the Hamiltonian operator [45],

$$
\hat{H}=\mathcal{N}_{a} \hat{\mathcal{H}}_{a}+\mathcal{N}_{[a b]} \hat{\mathcal{J}}_{[a b]},
$$

where $\mathcal{N}_{a}$ and $\mathcal{N}_{[a b]}$ are Lagrange multipliers, and

$$
\begin{aligned}
\hat{\mathcal{H}}_{a} & =\frac{1}{2}\left(\hat{P}_{a b c} \hat{P}_{b d e} \hat{M}_{c d e}-\lambda \hat{M}_{a b b}+i \lambda_{H} \hat{P}_{a b b}\right), \quad \lambda_{H}=\frac{(N+2)(N+3)}{12} \\
\hat{\mathcal{J}}_{[a b]} & =\frac{1}{4}\left(\hat{P}_{a c d} \hat{M}_{b c d}-\hat{P}_{b c d} \hat{M}_{a c d}\right) .
\end{aligned}
$$

Here summing over repeated indices is implied, while the symbol $[a b]$ refers to antisymmetry of indices such that $\hat{\mathcal{J}}_{[a b]}=-\hat{\mathcal{J}}_{[b a]}$. Ingredients appearing in (2.13) are explained in order: $\hat{\mathcal{J}}_{[a b]}$ serve as the generator of the $O(N)$-kinematical symmetry, $(2.7)$, and $\hat{\mathcal{H}}_{a}$ is the generator of the symmetry analogous to the temporal diffeomorphism in general relativity; they are first class constraints and form a closed constraint algebra:

$$
\begin{aligned}
{\left[\hat{\mathcal{H}}\left(\xi^{1}\right), \hat{\mathcal{H}}\left(\xi^{2}\right)\right] } & =\frac{i}{6} \hat{\mathcal{J}}\left(\left[\hat{\xi}^{1}, \hat{\xi^{2}}\right]+2 \lambda\left[\xi^{1}, \xi^{2}\right]\right), \\
{[\hat{\mathcal{J}}(\eta), \hat{\mathcal{H}}(\xi)] } & =\frac{i}{6} \hat{\mathcal{H}}(\eta \xi), \\
{\left[\hat{\mathcal{J}}\left(\eta^{1}\right), \hat{\mathcal{J}}\left(\eta^{2}\right)\right] } & =\frac{i}{6} \hat{\mathcal{J}}\left(\left[\eta^{1}, \eta^{2}\right]\right),
\end{aligned}
$$

where $\hat{\mathcal{H}}(\xi)=\xi_{a} \hat{\mathcal{H}}_{a}, \hat{\mathcal{J}}(\eta)=\eta_{[a b]} \hat{\mathcal{J}}_{[a b]}$ and $\hat{\xi}_{a b}=\hat{P}_{a b c} \xi_{c} ;[$,$] denotes the matrix commutator$ for $\hat{\xi}^{i}$, and $\left[\xi^{1}, \xi^{2}\right]_{[a b]}=\xi_{a}^{1} \xi_{b}^{2}-\xi_{a}^{2} \xi_{b}^{1}$. Note that this is not a Lie algebra with structure constants, but has non-linear structures as on the right-hand side of the first line. Following the standard nomenclature, we call $\hat{\mathcal{J}}_{[a b]}$ and $\hat{\mathcal{H}}_{a}$ momentum and Hamiltonian constraints, respectively. $\lambda_{H}$ is a real constant introduced by the operator ordering and has been fixed by imposing the hermiticity of $\hat{\mathcal{H}}_{a}$, while $\lambda$ is a real undetermined constant which we call cosmological constant. The last physical naming can be justified by its role in comparison with the mini-superspace approximation of general relativity [46]. It is worth to recall that, in the case of hermite tensors (2.8), the cosmological constant term is prohibited by the consistency of the constraint algebra [40], while this term is allowed for the minimal case as above.

In the case when $\lambda=0$, one can consistently incorporate the dilation generator to the Hamiltonian operator as $\hat{H} \rightarrow \hat{H}+\mathcal{N} \hat{\mathcal{D}}$, where $\mathcal{N}$ is the Lagrange multiplier and $\hat{\mathcal{D}}$ in operator form is given by [41],

$$
\hat{\mathcal{D}}=\frac{1}{6}\left(\hat{P}_{a b c} \hat{M}_{a b c}+i \lambda_{D}\right) .
$$

Here $\lambda_{D}$ is a constant introduced by the operator ordering, and can be fixed as

$$
\lambda_{D}=\frac{N(N+1)(N+2)}{12}
$$


by imposing the hermiticity of $\hat{\mathcal{D}}$. The constraint algebra remains closed even with the inclusion of the dilation generator, as can be seen for $\lambda=0$ from the following,

$$
\begin{aligned}
{[\hat{\mathcal{D}}, \hat{\mathcal{H}}(\xi)] } & =\frac{i}{6} \hat{\mathcal{H}}(\xi), \\
{[\hat{\mathcal{D}}, \hat{\mathcal{J}}(\eta)] } & =0 .
\end{aligned}
$$

However, we will not use this dilation generator as a genuine generator but merely as a mathematical tool for simplifying the process to find a solution to the constraint equations introduced below.

In the following sections, we will find the physical states satisfying the constraints,

$$
\hat{\mathcal{H}}_{a} \Psi=\hat{\mathcal{J}}_{[a b]} \Psi=0 .
$$

Note that here we do not impose the $\hat{\mathcal{D}}$ constraint to define the physical states. For later convenience, we introduce the $M$-representation of $\hat{P}_{a b c}$ and the $P$-representation of $\hat{M}_{a b c}$ as follows:

$$
\begin{aligned}
\hat{P}_{a b c}= & -i D_{a b c}^{M}=-i \Delta(a b c) \frac{\partial}{\partial M_{a b c}}, \quad \hat{M}_{a b c}=i D_{a b c}^{P}=i \Delta(a b c) \frac{\partial}{\partial P_{a b c}}, \\
\Delta(a b c)= & \left\{\begin{array}{l}
1, \text { for } a=b=c, \\
\frac{1}{3}, \text { for } a=b \neq c, b=c \neq a, c=a \neq b, \\
\frac{1}{6}, \text { for } a \neq b, b \neq c, c \neq a,
\end{array}\right.
\end{aligned}
$$

where $M$ and $P$ are the eigenvalues of $\hat{M}$ and $\hat{P}$, respectively, and $D_{a b c}^{M, P}$ are rescaled partial differentials satisfying

$$
D_{a b c}^{M} M_{d e f}=D_{a b c}^{P} P_{d e f}=\frac{1}{6} \sum_{\sigma} \delta_{a \sigma(d)} \delta_{b \sigma(e)} \delta_{c \sigma(f)} .
$$

Finally, let us introduce a classification of the physical states in two types in the case with no cosmological constant, $\lambda=0$. In this case, from (2.14), the Hamiltonian constraints can be rewritten in a form,

$$
\hat{\mathcal{H}}_{a}=\frac{1}{2} \hat{P}_{a b c} \hat{\mathcal{J}}_{(b c)}
$$

where

$$
\hat{\mathcal{J}}_{(a b)}=\frac{1}{2}\left(\hat{P}_{a c d} \hat{M}_{b c d}+\hat{P}_{b c d} \hat{M}_{a c d}\right)+i \lambda_{H} \delta_{a b}
$$

with the round brackets of $(a b)$ symbolically representing the symmetric feature of the two indices. Then, the solutions to the constraints can be classified into the following two types,

$$
\begin{aligned}
\text { Kinematical: } & \hat{\mathcal{J}}_{(a b)} \Psi=\hat{\mathcal{J}}_{[a b]} \Psi=0, \\
\text { Dynamical: } & \hat{\mathcal{H}}_{a} \Psi=\hat{\mathcal{J}}_{[a b]} \Psi=0,{ }^{\exists} \hat{\mathcal{J}}_{(a b)} \Psi \neq 0,
\end{aligned}
$$


The naming of "kinematical" comes from the fact that the operators, $\hat{\mathcal{J}}_{(a b)}$ and $\hat{\mathcal{J}}_{[a b]}$, form a $\operatorname{gl}(N)$ Lie algebra. This is a linear Lie algebra with structure constants, and such kinematical physical states would reflect only the kinematical characters rather than the dynamics of the canonical tensor model. A naive expectation is that physically interesting dynamics is caused by the non-linear features of the constraint algebra (2.16) with structure functions, ${ }^{5}$ as in general relativity. From this viewpoint, the dynamical states would be of more importance. From (2.24), a necessary condition for a kinematical state, which is a convenient criterion in subsequent analysis, is given by

$$
\left[\hat{P}_{a b c} \hat{M}_{a b c}+i N \lambda_{H}\right] \Psi=0
$$

In the $P$ and $M$ representations, this criterion is respectively represented as

$$
\begin{aligned}
\left(P_{a b c} D_{a b c}^{P}+N \lambda_{H}\right) \Psi(P) & =0, \\
{\left[M_{a b c} D_{a b c}^{M}+\frac{(N-1) N(N+2)}{12}\right] \Psi(M) } & =0 .
\end{aligned}
$$

\section{$3 \quad N=2$ model}

In this section we will consider the case of $N=2$ tensor model both with and without a cosmological constant. In either case, we will explicitly solve the constraint equations (2.20) to find the physical wave-function.

\subsection{Case without a cosmological constant}

In this subsection we consider the $N=2$ canonical tensor model with no cosmological constant, thereby setting $\lambda=0$. In this model, in the $P$-representation, there are 4 independent variables, $\left\{P_{111}, P_{112}, P_{122}, P_{222}\right\}$. On the other hand, the constraint equations $(2.20)$ give only three independent first order partial differential equations:

$$
\hat{\mathcal{H}}_{1} \Psi(P)=\hat{\mathcal{H}}_{2} \Psi(P)=\hat{\mathcal{J}}_{[12]} \Psi(P)=0 .
$$

This implies that the most general solution to (3.1) can be written with an arbitrary function of a single variable. In order to find this, we first consider a solution on a twodimensional subspace, and then write it in an $O(2)$-invariant form to extend it on the whole space.

We start by introducing a 2-dimensional subspace (or a gauge choice with respect to $\mathcal{J}_{[12]}$ and $\mathcal{D}$ ) in the $P$-representation:

$$
P_{111}=1, \quad P_{112}=0, \quad P_{122}=x_{1}, \quad P_{222}=x_{2},
$$

\footnotetext{
${ }^{5}$ It would be more appropriate to call them "structure operators", since we are considering the quantized case.
} 
where $x_{1}, x_{2}$ are the variables parameterizing the subspace. In this 2-dimensional subspace, the constraint equations (3.1) are given by

$$
\begin{aligned}
\hat{\mathcal{H}}_{1} \Psi & \propto\left[3 \frac{\partial}{\partial P_{111}}+x_{1}\left(1+2 x_{1}\right) \frac{\partial}{\partial x_{1}}+3 x_{1} x_{2} \frac{\partial}{\partial x_{2}}+5\left(1+x_{1}\right)\right] \Psi=0, \\
\hat{\mathcal{H}}_{2} \Psi & \propto\left[x_{1}\left(1+2 x_{1}\right) \frac{\partial}{\partial P_{112}}+3 x_{1} x_{2} \frac{\partial}{\partial x_{1}}+3\left(x_{1}^{2}+x_{2}^{2}\right) \frac{\partial}{\partial x_{2}}+5 x_{2}\right] \Psi=0, \\
\hat{\mathcal{J}}_{[12]} \Psi & \propto\left[\left(1-2 x_{1}\right) \frac{\partial}{\partial P_{112}}-x_{2} \frac{\partial}{\partial x_{1}}+3 x_{1} \frac{\partial}{\partial x_{2}}\right] \Psi=0 .
\end{aligned}
$$

By using (3.5), we remove $\partial / \partial P_{112}$ from (3.4), which is a direction straying away from the subspace (3.2). This gives us the following equation,

$$
\left[4 x_{1} x_{2}\left(x_{1}-1\right) \frac{\partial}{\partial x_{1}}+3\left(4 x_{1}^{3}+2 x_{1} x_{2}^{2}-x_{2}^{2}\right) \frac{\partial}{\partial x_{2}}+5 x_{2}\left(2 x_{1}-1\right)\right] \Psi=0 .
$$

In fact, a solution to (3.6) has been found in [45]: ${ }^{6}$

$$
\Psi=\frac{\sqrt{4 x_{1}^{3}+x_{2}^{2}}}{x_{1}^{2}\left(x_{1}-1\right)^{2}}
$$

Then, the general solution to (3.6) is given by

$$
\Psi=f\left(x_{1}, x_{2}\right) \frac{\sqrt{4 x_{1}^{3}+x_{2}^{2}}}{x_{1}^{2}\left(x_{1}-1\right)^{2}},
$$

where $f\left(x_{1}, x_{2}\right)$ satisfies the homogeneous part of (3.6), i.e.,

$$
\left[4 x_{1} x_{2}\left(x_{1}-1\right) \frac{\partial}{\partial x_{1}}+3\left(4 x_{1}^{3}+2 x_{1} x_{2}^{2}-x_{2}^{2}\right) \frac{\partial}{\partial x_{2}}\right] f=0 .
$$

This implies that $f$ is constant along the characteristics,

$$
\frac{d x_{2}}{d x_{1}}=\frac{3\left(4 x_{1}^{3}+2 x_{1} x_{2}^{2}-x_{2}^{2}\right)}{4 x_{1} x_{2}\left(x_{1}-1\right)}
$$

This can be solved to obtain,

$$
c_{0}=\frac{\left(4 x_{1}^{3}+x_{2}^{2}\right)^{4}}{x_{1}{ }^{6}\left(1-x_{1}\right)^{6}}
$$

where $c_{0}$ is a constant. Therefore, we get the following result,

$$
f\left(x_{1}, x_{2}\right)=g\left(\frac{\left(4 x_{1}^{3}+x_{2}^{2}\right)^{4}}{x_{1}^{6}\left(1-x_{1}\right)^{6}}\right)
$$

where $g(z)$ is an arbitrary function of $z$. This solution can be written in an $O(2)$-invariant form. As demonstrated in [45], on the 2-dimensional subspace (3.2), one can explicitly check that

$$
\begin{aligned}
& A(P)=\epsilon_{a c} \epsilon_{b d} \epsilon_{e g} \epsilon_{f h} \epsilon_{e^{\prime} g^{\prime}} \epsilon_{f^{\prime} h^{\prime}} P_{a e f} P_{b g h} P_{c e^{\prime} f^{\prime}} P_{d g^{\prime} h^{\prime}}=-2\left(4 x_{1}{ }^{3}+x_{2}{ }^{2}\right), \\
& B(P)=P_{a c d} P_{b d e} P_{b e f} P_{a f c}-P_{a c d} P_{b d e} P_{a e f} P_{b f c}=2\left(1-x_{1}\right)^{2} x_{1}{ }^{2},
\end{aligned}
$$

\footnotetext{
${ }^{6}$ This is the exact solution of the $N=2$ canonical tensor model incorporating also the dilation constraint (2.17). However, (3.7) of course satisfies (3.6) in the present case.
} 
where $\epsilon_{12}=-\epsilon_{21}=1, \epsilon_{11}=\epsilon_{22}=0$. Therefore, one can extend the solution above to an $O(2)$-invariant form:

$$
\Psi(P)=g\left(\frac{A(P)^{4}}{B(P)^{3}}\right) \frac{\sqrt{A(P)}}{B(P)} .
$$

In fact, one can explicitly check that (3.15) solves (3.1) on the whole space. Since the solution (3.15) contains an arbitrary function of a single variable, $g(z)$, it should be the most general solution to (3.1).

Let us classify the general solution (3.15) into the kinematical and dynamical solutions discussed in the last of section 2. The necessary criterion (2.28) for a kinematical solution requires that a wave-function be a homogenous function of $P$ with degree $-N \lambda_{H}=-\frac{10}{3}$. This determines $g(z)=z^{-\frac{1}{3}}$, and therefore,

$$
\Psi_{\text {kin }}(P)=A(P)^{-\frac{5}{6}}
$$

In fact, one can explicitly check that, not only the necessary criterion, but also the full conditions for a kinematical state, $\hat{\mathcal{J}}_{(a b)} \Psi_{\text {kin }}=0$, are satisfied by (3.16). Then, the other wave-functions than (3.16) are the dynamical ones. This in turn implies that the potential singularities at $B(P)=0$ of the general wave-function (3.15) are purely of dynamical origin. As was argued in [45], the configurations satisfying $B(P)=0$ are those of maximal locality. This would mean that the non-linear character of the constraints is essentially important in the emergence of locality in the canonical tensor model.

The characteristics of the configurations satisfying $A(P)=0$, which are of kinematical origin as shown above, can be discussed in terms of the fuzzy space interpretation presented in section 2. Let us consider the following condition:

$$
v_{a} v_{b} P_{a b c}=0,
$$

where $v$ is a non-vanishing vector. Then, in the gauge (3.2), one can easily show that such a non-vanishing $v$ exists, if and only if $4 x_{1}^{3}+x_{2}^{2}=0$, which exactly corresponds to $A(P)=0$. In the fuzzy space interpretation of the canonical tensor model explained in section 2 , the "points" in a fuzzy space, $f_{a}$ 's, are assumed to form an algebra, ${ }^{7}$

$$
f_{a} \star f_{b}=P_{a b c} f_{c}
$$

Thus the condition (3.17) implies that a "point", $f=v_{a} f_{a}$, satisfies

$$
f \star f=0 .
$$

Therefore, $A(P)=0$ has the meaning that there exists a "point", $f$, with the property of a Grassmann number. We will see in section 4.1 that configurations with the same nature appear as potential singularities also in the case of $N=3$.

\footnotetext{
${ }^{7}$ Here we do not care about which of $M$ or $P$ defines the fuzzy space algebra. At present, we have no good argument to determine which choice is more proper.
} 


\subsection{Case with a cosmological constant}

We now consider the $N=2$ tensor model with a cosmological constant. Since the corresponding constraint equations are more involved and finding the most general solution does not seem straightforward on first sight, we will first solve them on a subspace invariant under a $Z_{2}$ transformation. We will then extend the solution over the whole space by finding an $O(2)$ invariant expression. The reason why we consider in this subsection such a symmetric subspace rather than (3.2) is that the equations are simplified because of the symmetry and a similar procedure as below can also be applied to the case of $N=3$ as in section 4 .

The subspace we consider is parameterized by

$$
P_{111}=P_{222}=y_{1}, \quad P_{112}=P_{221}=y_{2},
$$

which are the fixed points of the $Z_{2}$ transformation permuting the index set, $\{1,2\}$. The method of characteristics employed in section 3.1 reduces the problem of solving the set of the first-order partial differential equations representing the constraints to solving the ordinary first-order differential equations along the flows generated by the constraints. An infinitesimal variation of $\hat{P}$ generated by the constraints is given by

$$
\delta \hat{P}_{a b c}=i\left[\hat{P}_{a b c}, \xi_{d} \hat{\mathcal{H}}_{d}+\eta_{[d e]} \hat{\mathcal{J}}_{[d e]}\right],
$$

where $\xi$ and $\eta$ are infinitesimal parameters. One can easily show that the infinitesimal variation (3.21) goes out of the subspace (3.20), unless we require

$$
\xi_{1}=\xi_{2}=\xi, \quad \eta_{[a b]}=0 .
$$

This requirement in turn determines the constraint equations on the $Z_{2}$ subspace as

$$
\left(\hat{\mathcal{H}}_{1}+\hat{\mathcal{H}}_{2}\right) \Psi_{\lambda}=0
$$

where $\Psi_{\lambda}$ is an wave-function. By noting that

$$
\frac{\partial}{\partial y_{1}}=\sum_{a=1}^{2} \frac{\partial}{\partial P_{a a a}}, \quad \frac{\partial}{\partial y_{2}}=\sum_{\substack{a, b=1 \\ a \neq b}}^{2} \frac{\partial}{\partial P_{a b b}},
$$

Eq. (3.23) becomes

$$
\left[3\left(y_{1}^{2}+2 y_{2}^{2}+y_{1} y_{2}-\lambda\right) \frac{\partial}{\partial y_{1}}+\left(7 y_{2}^{2}+5 y_{1} y_{2}-\lambda\right) \frac{\partial}{\partial y_{2}}+10\left(y_{1}+y_{2}\right)\right] \Psi_{\lambda}=0 .
$$

From the result of section 3.1, we know that the solution for $\lambda=0$ is given by (3.15) with

$$
\begin{aligned}
& A(y)=-2\left(y_{1}+3 y_{2}\right)\left(y_{1}-y_{2}\right)^{3}, \\
& B(y)=\left[2 \sqrt{2}\left(y_{1}-y_{2}\right) y_{2}\right]^{2},
\end{aligned}
$$


where $A(y), B(y)$ have been obtained by putting (3.20) to (3.13) and (3.14), respectively. In fact, $A(y), B(y)$ satisfy the following peculiar properties,

$$
\begin{aligned}
& \mathcal{O}_{0} A(y)=12\left(y_{1}+y_{2}\right) A(y), \\
& \mathcal{O}_{0} B(y)=16\left(y_{1}+y_{2}\right) B(y),
\end{aligned}
$$

where $\mathcal{O}_{0}=\mathcal{O}_{\lambda=0}$ with $\mathcal{O}_{\lambda}$ defined by the derivative part of (3.25),

$$
\mathcal{O}_{\lambda}=3\left(y_{1}^{2}+2 y_{2}^{2}+y_{1} y_{2}-\lambda\right) \frac{\partial}{\partial y_{1}}+\left(7 y_{2}^{2}+5 y_{1} y_{2}-\lambda\right) \frac{\partial}{\partial y_{2}} .
$$

This means that (3.25) with $\lambda=0$ can be rewritten as a partial differential equation with variables $A, B$ rather than $y_{1}, y_{2}$, because, from the Leibniz rule,

$$
\begin{aligned}
{\left[\mathcal{O}_{0}+10\left(y_{1}+y_{2}\right)\right] \Psi(y) } & =\left[\left(\mathcal{O}_{0} A\right) \frac{\partial}{\partial A}+\left(\mathcal{O}_{0} B\right) \frac{\partial}{\partial B}+10\left(y_{1}+y_{2}\right)\right] \Psi(A, B) \\
& =\left(y_{1}+y_{2}\right)\left[12 A \frac{\partial}{\partial A}+16 B \frac{\partial}{\partial B}+10\right] \Psi(A, B)=0 .
\end{aligned}
$$

When $A, B$ are regarded as variables, the expression (3.15) is indeed the most general solution to the simple partial differential equation in the last line. Therefore, if we keep the properties (3.28) and (3.29) for general $\lambda$, the solution to (3.25) is simply given by the same expression as (3.15) with proper replacements of $A(y)$ and $B(y)$. Namely, the solution is given by

$$
\Psi_{\lambda}(y)=g\left(\frac{A_{\lambda}(y)^{4}}{B_{\lambda}(y)^{3}}\right) \frac{\sqrt{A_{\lambda}(y)}}{B_{\lambda}(y)},
$$

where $g(z)$ is an arbitrary function, and $A_{\lambda}(y), B_{\lambda}(y)$ must be determined through

$$
\frac{\mathcal{O}_{\lambda} A_{\lambda}(y)}{A_{\lambda}(y)}=\frac{\mathcal{O}_{0} A(y)}{A(y)} \text {, similarly for } B_{\lambda}(y) \text {. }
$$

It does not seem always guaranteed that one can obtain the solutions of $A_{\lambda}(y), B_{\lambda}(y)$ to (3.33) in simple expressions. But, rather miraculously, we obtain

$$
\begin{aligned}
& A_{\lambda}(y)=-2\left(y_{1}+3 y_{2}\right)\left(y_{1}-y_{2}\right)^{3}+4 \lambda\left(y_{1}+y_{2}\right)^{2}-20 \lambda\left(y_{1}-y_{2}\right) y_{2}-\lambda^{2}, \\
& B_{\lambda}(y)=\left[2 \sqrt{2}\left(y_{1}-y_{2}\right) y_{2}-\frac{\sqrt{2}}{4} \lambda\right]^{2} .
\end{aligned}
$$

The reason why we get such simple expressions even for general $\lambda$ is probably related with what will be discussed in section 11 .

The solution in the whole space can simply be obtained by finding the $O(2)$-invariant expressions of $A_{\lambda}(y), B_{\lambda}(y)$ :

$$
\begin{aligned}
& \Psi_{\lambda}(P)=g\left(\frac{A_{\lambda}(P)^{4}}{B_{\lambda}(P)^{3}}\right) \frac{\sqrt{A_{\lambda}(P)}}{B_{\lambda}(P)}, \\
& A_{\lambda}(P)=I_{2}(P)-5 \sqrt{2} \lambda I_{1}(P)+2 \lambda I_{3}(P)-\lambda^{2}, \\
& B_{\lambda}(P)=\left[I_{1}(P)-\frac{\sqrt{2}}{4} \lambda\right]^{2},
\end{aligned}
$$


where

$$
\begin{aligned}
& I_{1}(P)=\frac{1}{\sqrt{2}} \epsilon_{i_{1} i_{2}} \epsilon_{i_{3} i_{4}} P_{i_{1} i_{3} j} P_{i_{2} i_{4} j}, \\
& I_{2}(P)=\epsilon_{j_{1} l_{1}} \epsilon_{j_{2} l_{2}} \epsilon_{i_{1} i_{2}} \epsilon_{i_{3} i_{4}} P_{i_{1} i_{3} j_{1}} P_{i_{2} i_{4} j_{2}} \epsilon_{k_{1} k_{2}} \epsilon_{k_{3} k_{4}} P_{k_{1} k_{3} l_{1}} P_{k_{2} k_{4} l_{2}}, \\
& I_{3}(P)=P_{i j j} P_{i k k} .
\end{aligned}
$$

In fact, one can explicitly check this is the solution in the whole space.

\section{$4 \quad N=3$ model}

In this section we extend our horizons further and explore the $N=3$ tensor model both with and without a cosmological constant. While, in this more complicated model, it is hard to find the general solution to the constraint equations (2.20), it is possible to find the most general solution in a subspace satisfying a $Z_{3}$ symmetry. The procedure is basically the same as that employed for $N=2$ in section 3.2 .

\subsection{Case with no cosmological constant}

Here we consider the $N=3$ minimal tensor model with no cosmological constant, and find the general solution to the constraint equations on a subspace invariant under a $Z_{3}$ symmetry. The subspace is parametrized by

$$
P_{a a a}=x_{1}, \quad P_{a b b}=x_{2}, \quad P_{a b c}=x_{3},
$$

for any $a \neq b \neq c \neq a$, which are the fixed points of the $Z_{3}$ transformations permuting the index set $\{1,2,3\}$. As in section 3.2 , one can show that there exists only one linear combination of the constraints which generates a flow along the $Z_{3}$ symmetric subspace (4.1), and it is given by

$$
\hat{\mathcal{C}}^{(3)}=\sum_{a=1}^{3} \hat{\mathcal{H}}_{a}
$$

On the subspace (4.1), the constraint (4.2) is expressed as

$$
\begin{aligned}
\hat{\mathcal{C}}^{(3)}= & \frac{i}{2}\left(x_{1}^{2}+2 x_{1} x_{2}+2 x_{2}\left(2 x_{2}+x_{3}\right)\right) \frac{\partial}{\partial x_{1}} \\
& +\frac{i}{6}\left(5 x_{1} x_{2}+12 x_{2}^{2}+x_{1} x_{3}+7 x_{2} x_{3}+2 x_{3}^{2}\right) \frac{\partial}{\partial x_{2}} \\
& +\frac{i}{2}\left(x_{1} x_{3}+4 x_{2}\left(x_{2}+x_{3}\right)\right) \frac{\partial}{\partial x_{3}}+\frac{3}{2} i \lambda_{H}\left(x_{1}+2 x_{2}\right),
\end{aligned}
$$

where $\lambda_{H}=\frac{5}{2}$, and we have used

$$
\frac{\partial}{\partial x_{1}}=\sum_{a=1}^{3} \frac{\partial}{\partial P_{a a a}}, \quad \frac{\partial}{\partial x_{2}}=\sum_{\substack{a, b=1 \\ a \neq b}}^{3} \frac{\partial}{\partial P_{a b b}}, \quad \frac{\partial}{\partial x_{3}}=\frac{\partial}{\partial P_{123}} .
$$


In order to further simplify the process of finding a solution, let us virtually impose the dilation constraint (2.17):

$$
\hat{\mathcal{D}}=\frac{i}{6}\left(\sum_{i=1}^{3} x_{i} \frac{\partial}{\partial x_{i}}+\lambda_{D}\right),
$$

where $\lambda_{D}$ is supposed to be a free parameter for the purpose, rather than the fixed value in $(2.18)$.

By assuming the two constraints, (4.3) and (4.5), and $\lambda_{D}=0$ for the time being, we can eliminate $\partial / \partial x_{1}$ to obtain a constraint,

$$
\begin{aligned}
\hat{\mathcal{C}}_{v}^{(3)}= & \frac{i}{6 x_{1}}\left(2 x_{2}+x_{3}\right)\left\{\left(x_{1}^{2}+3 x_{1} x_{2}-6 x_{2}^{2}+2 x_{1} x_{3}\right) \frac{\partial}{\partial x_{2}}+6 x_{2}\left(x_{1}-x_{3}\right) \frac{\partial}{\partial x_{3}}\right\} \\
& +\frac{3}{2} i \lambda_{H}\left(x_{1}+2 x_{2}\right) .
\end{aligned}
$$

The method of characteristics applied to this partial differential equation, $\hat{\mathcal{C}}_{v}^{(3)} \Psi=0$, reduces the problem to solving

$$
\begin{aligned}
d s=\frac{d x_{2}}{\left(2 x_{2}+x_{3}\right)\left(x_{1}^{2}+3 x_{1} x_{2}-6 x_{2}^{2}+2 x_{1} x_{3}\right)} & =\frac{d x_{3}}{6 x_{2}\left(2 x_{2}+x_{3}\right)\left(x_{1}-x_{3}\right)}, \\
\frac{d \Psi}{\Psi}+9 \lambda_{H} x_{1}\left(x_{1}+2 x_{2}\right) d s & =0,
\end{aligned}
$$

where $s$ parametrizes the trajectory of the flow. Note that $x_{1}$ is regarded as a constant in these equations. The trajectory equation derived from (4.7),

$$
\frac{d x_{2}}{d x_{3}}=\frac{x_{1}^{2}+3 x_{1} x_{2}-6 x_{2}^{2}+2 x_{1} x_{3}}{6 x_{2}\left(x_{1}-x_{3}\right)}
$$

tells us that the following combination remains unchanged along the trajectory:

$$
\frac{\left(-x_{1}+x_{3}\right)^{3}}{\left(-x_{1}+3 x_{2}-2 x_{3}\right)^{2}\left(x_{1}+6 x_{2}+2 x_{3}\right)} \text {. }
$$

This gives a motivation to consider an ansatz:

$$
\Psi=\frac{\left(-x_{1}+x_{3}\right)^{c_{1}}\left(2 x_{2}+x_{3}\right)^{c_{2}}}{\left(-x_{1}+3 x_{2}-2 x_{3}\right)^{c_{3}}\left(x_{1}+6 x_{2}+2 x_{3}\right)^{c_{4}}},
$$

where $\left(2 x_{2}+x_{3}\right)$ can be read off from (4.7), and $c_{i}$ 's are numerical constants to be determined. Inserting (4.11) into the equations, $\hat{\mathcal{C}}^{(3)} \Psi=\hat{\mathcal{D}} \Psi=0$, one obtains

$$
\begin{aligned}
c_{2} & =\frac{3}{2}\left(\lambda_{D}-3 \lambda_{H}\right), \\
c_{3} & =\frac{2}{3} c_{1}+\lambda_{D}-\lambda_{H}, \\
c_{4} & =\frac{1}{3} c_{1}+\frac{1}{2}\left(3 \lambda_{D}-7 \lambda_{H}\right) .
\end{aligned}
$$


Since $c_{1}$ and $\lambda_{D}$ can be taken arbitrary to satisfy the single constraint equation, $\hat{\mathcal{C}}^{(3)} \Psi=0$, the general form of $\Psi$ is determined to be

$$
\begin{aligned}
\Psi= & \left(-x_{1}+3 x_{2}-2 x_{3}\right)^{\lambda_{H}}\left(2 x_{2}+x_{3}\right)^{-\frac{9}{2} \lambda_{H}}\left(x_{1}+6 x_{2}+2 x_{3}\right)^{\frac{7}{2} \lambda_{H}} \\
& \times f\left(\frac{\left(2 x_{2}+x_{3}\right)^{3}}{\left(-x_{1}+3 x_{2}-2 x_{3}\right)^{2}\left(x_{1}+6 x_{2}+2 x_{3}\right)^{3}}, \frac{\left(-x_{1}+x_{3}\right)^{3}}{\left(-x_{1}+3 x_{2}-2 x_{3}\right)^{2}\left(x_{1}+6 x_{2}+2 x_{3}\right)}\right),
\end{aligned}
$$

where $f$ is an arbitrary function with two arguments. It is clear from number counting that (4.15) gives the most general solution to the constraint equation, $\hat{\mathcal{C}}^{(3)} \Psi=0$.

Let us study peaks of the wave-function in (4.11). Since we cannot uniquely determine the coefficients, $c_{i}$, here we consider possible peaks of the wave function (4.11) assuming signs of the coefficients. Firstly, if $c_{3}>0$, then (4.11) diverges when $-x_{1}+3 x_{2}-2 x_{3}=$ 0 . This means that the configurations satisfying this equation are enhanced. In fact, it can be shown that, if $-x_{1}+3 x_{2}-2 x_{3}=0, P_{a b c}$ is invariant under an infinitesimal $\mathrm{SO}(2)$ transformation:

$$
t_{a a^{\prime}} P_{a^{\prime} b c}+t_{b b^{\prime}} P_{a b^{\prime} c}+t_{c c^{\prime}} P_{a b c^{\prime}}=0
$$

where

$$
t=\left(\begin{array}{ccc}
0 & 1 & -1 \\
-1 & 0 & 1 \\
1 & -1 & 0
\end{array}\right) .
$$

Therefore, the configurations with the $\mathrm{SO}(2)$ symmetry are enhanced in the wave-function.

Secondly, if $c_{4}>0$, then the wave-function (4.36) diverges when $x_{1}+6 x_{2}+2 x_{3}=0$. This configuration satisfies

$$
v_{a} v_{b} P_{a b c}=x_{1}+6 x_{2}+2 x_{3}=0
$$

where

$$
v=(1,1,1)
$$

This is the same situation discussed for the case of $N=2$ in the last paragraph of section 3.1. Therefore, the condition, $x_{1}+6 x_{2}+2 x_{3}=0$, has the meaning that there exists a "point", $f=v_{a} f_{a}$, with the property of a Grassmann number.

A more interesting possibility arises for the configurations which simultaneously satisfy the two conditions above, $-x_{1}+3 x_{2}-2 x_{3}=x_{1}+6 x_{2}+2 x_{3}=0$. They are the configurations satisfying

$$
x_{2}=0, \quad x_{3}=-\frac{x_{1}}{2} .
$$

What is interesting on these points is that, in addition to the $\mathrm{SO}(2)$ symmetry mentioned above, $P_{a b c}$ is invariant under an infinitesimal boost transformation,

$$
k_{a a^{\prime}} P_{a^{\prime} b c}+k_{b b^{\prime}} P_{a b^{\prime} c}+k_{c c^{\prime}} P_{a b c^{\prime}}=0
$$


where

$$
k=\left(\begin{array}{lll}
0 & 1 & 1 \\
1 & 0 & 1 \\
1 & 1 & 0
\end{array}\right) .
$$

Therefore, these configurations are invariant under a Lorentz group. In the present $N=3$ case, the generators commute with each other, $[k, t]=0$, and may not be of much interest. However, we expect enhancement of non-abelian Lorentz groups to occur for larger $N$.

We could not find similar kinematical characterization as above for the other possible peaks at $2 x_{2}+x_{3}=0$ or $-x_{1}+x_{3}=0$. Probably, these are more rooted in dynamics, similarly to $B(P)=0$ for $N=2$ as discussed in section 3.1.

\subsection{Case with a cosmological constant}

In this subsection, we generalize the solution on the $Z_{3}$ symmetric subspace obtained in section 4.1 to include the cosmological constant $\lambda$ by applying the method employed in section 3.2 for the case of $N=2$. With $\lambda$, the constraint (4.3) is replaced by

$$
\begin{aligned}
\hat{\mathcal{C}}_{\lambda}^{(3)}= & \sum_{a=1}^{3} \hat{\mathcal{H}}_{a} \\
= & \frac{i}{2}\left(-\lambda+x_{1}{ }^{2}+2 x_{1} x_{2}+2 x_{2}\left(2 x_{2}+x_{3}\right)\right) \frac{\partial}{\partial x_{1}} \\
& +\frac{i}{6}\left(-\lambda+5 x_{1} x_{2}+12 x_{2}{ }^{2}+x_{1} x_{3}+7 x_{2} x_{3}+2 x_{3}{ }^{2}\right) \frac{\partial}{\partial x_{2}} \\
& +\frac{i}{2}\left(x_{1} x_{3}+4 x_{2}\left(x_{2}+x_{3}\right)\right) \frac{\partial}{\partial x_{3}}+\frac{3}{2} i \lambda_{H}\left(x_{1}+2 x_{2}\right) .
\end{aligned}
$$

As in section 3.2, let us take the derivative part of the constraint (4.23) as

$$
\begin{aligned}
\mathcal{O}_{\lambda}^{(3)}= & \frac{i}{2}\left(-\lambda+x_{1}^{2}+2 x_{1} x_{2}+2 x_{2}\left(2 x_{2}+x_{3}\right)\right) \frac{\partial}{\partial x_{1}} \\
& +\frac{i}{6}\left(-\lambda+5 x_{1} x_{2}+12 x_{2}{ }^{2}+x_{1} x_{3}+7 x_{2} x_{3}+2 x_{3}{ }^{2}\right) \frac{\partial}{\partial x_{2}} \\
& +\frac{i}{2}\left(x_{1} x_{3}+4 x_{2}\left(x_{2}+x_{3}\right)\right) \frac{\partial}{\partial x_{3}} .
\end{aligned}
$$

The solutions (4.15) for $\lambda=0$ are expressed in terms of the following linear combinations of $x_{i}$ 's:

$$
\begin{aligned}
E_{1} & =-x_{1}+3 x_{2}-2 x_{3}, \\
e_{2} & =2 x_{2}+x_{3}, \\
e_{3} & =x_{1}+6 x_{2}+2 x_{3}, \\
e_{4} & =-x_{1}+x_{3} .
\end{aligned}
$$

In the same way as the $N=2$ case, one would be able to construct solutions in the case of $\lambda \neq 0$ by considering corrections to these $E_{1}, e_{2,3,4}$ so that they satisfy the requirement 
corresponding to (3.33). In fact, $E_{1}$ satisfies

$$
\frac{\mathcal{O}_{\lambda}^{(3)} E_{1}}{E_{1}}=\frac{i}{2}\left(x_{1}-x_{3}\right) .
$$

The crucial point to note here is that the right-hand side of (4.29) is independent of $\lambda$, and therefore $E_{1}$ meets the requirement without any corrections.

Let us next consider $e_{3}$. In this case,

$$
\mathcal{O}_{\lambda}^{(3)} e_{3}=\frac{i}{2}\left(x_{1}+6 x_{2}+2 x_{3}\right) e_{3}-\frac{3}{2} i \lambda
$$

which requires us to make corrections to $e_{3}$. Since the dimension of $\lambda$ is that of $x_{i}{ }^{2}, e_{3}$ is not appropriate to consider corrections in perturbation of $\lambda$. Instead, we would be able to consider $e_{3}^{2}$ and its correction as

$$
E_{3}=e_{3}^{2}+\gamma \lambda
$$

where $\gamma$ is a numerical constant to be determined. Applying (4.24) on $E_{3}$, one obtains

$$
\mathcal{O}_{\lambda}^{(3)} E_{3}=i\left(x_{1}+6 x_{2}+2 x_{3}\right)\left(e_{3}^{2}-3 \lambda\right) .
$$

Therefore, if one takes $\gamma=-3$ in (4.31), then

$$
\frac{\mathcal{O}_{\lambda}^{(3)} E_{3}}{E_{3}}=i\left(x_{1}+6 x_{2}+2 x_{3}\right) \text {. }
$$

The righthand side does not contain $\lambda$, and therefore $E_{3}$ satisfies the requirement. In a similar manner, we can find suitable combinations of variables as

$$
\begin{aligned}
& E_{2}=e_{2} e_{4}+\frac{\lambda}{4}, \\
& E_{4}=e_{4}{ }^{3}+\frac{3}{4} \lambda\left(2 x_{1}-6 x_{2}-5 x_{3}\right) .
\end{aligned}
$$

Thus, using the new set of variables, $\left\{E_{1}, E_{2}, E_{3}, E_{4}\right\}$, one can generalize (4.15) to

$$
\Psi_{\lambda}=E_{1}{ }^{\lambda_{H}} E_{2}{ }^{-\frac{9}{2} \lambda_{H}} E_{3}{ }^{\frac{7}{4} \lambda_{H}} E_{4}{ }^{\frac{3}{2} \lambda_{H}} f\left(\frac{E_{2}^{3}}{E_{1}^{4} E_{3}{ }^{2}}, \frac{E_{4}{ }^{2}}{E_{1}^{4} E_{3}}\right)
$$

where $f\left(z_{1}, z_{2}\right)$ is an arbitrary function. In fact, one can explicitly check that (4.36) satisfies

$$
\hat{\mathcal{C}}_{\lambda}^{(3)} \Psi_{\lambda}=0 .
$$

As shown above for $N=3$, we have again found that, similar to the case of $N=2$, the inclusion of the cosmological constant can be implemented by making some corrections to the solutions with no cosmological constant. This suggests that the inclusion of the cosmological constant does not change the essential structure of the theory. In fact, we will see in section 11 that the cases with a cosmological constant can be treated by considering the cases with no cosmological constant for increased $N$. 


\section{Solutions in terms of statistical systems on random networks}

So far we have studied the solutions of the constraint equations of the canonical tensor model for the simple cases of $N=2,3$ by explicitly writing down and solving the sets of the partial differential equations representing the constraints. While this procedure can work for small $N$ 's, it is obvious that the situation becomes much more complicated as soon as one moves to higher- $N$ tensor model, and one quickly notices the limitations of the methodologies used in studying the $N=2,3$ cases. In the present and following sections, instead of giving general explicit solutions for particular $N$ 's, we will present some series of solutions valid for general $N$, by using the partition functions of statistical systems on random networks and some variants.

In [47], it was argued that the $N=2$ canonical tensor model is intimately related to the Ising model on random networks of trivalent vertices. In fact, it has been shown that the phase structure of the Ising model on random networks can be derived from the Hamiltonian vector flow of the canonical tensor model for $N=2$, if the flow is regarded as the renormalization group flow of the Ising model. The present and following sections will give further relations between the canonical tensor model and statistical systems on random networks by obtaining the physical wave-functions solving the constraints from the perspective of statistical systems on random networks.

Let us start with the following form of the "grand-type" partition function of statistical systems on random networks [47],

$$
Z\left(k, M, \mathcal{C}_{\phi}\right)=\int_{\mathcal{C}_{\phi}} d \phi e^{-k \phi^{2}+M \phi^{3}},
$$

where we have used shorthand notations,

$$
\begin{aligned}
d \phi & \equiv \prod_{a=1}^{N} \mathrm{~d} \phi_{a}, \\
\phi^{2} & \equiv \phi_{a} \phi_{a}, \\
M \phi^{3} & \equiv M_{a b c} \phi_{a} \phi_{b} \phi_{c} .
\end{aligned}
$$

Here $k$ and $M_{a b c}(a, b, c=1,2, \cdots, N)$ are a numerical variable and a three-index symmetric tensor, respectively. $\mathcal{C}_{\phi}$ denotes the domain of integration over $\phi_{a}$ 's, which can generally take complex values.

As discussed in [47], to make relations with statistical systems on random networks, $k$ is assumed to be a positive real number, and the integration contour, $\mathcal{C}_{\phi}$, is taken, for instance among various allowed possibilities, as

$$
\phi_{a}=e^{\frac{\pi i}{6}} r_{a}
$$

where $r_{a}$ run from $-\infty$ to $\infty$ on the real axis. By expanding the integrand of (5.1) in $M$, one obtains an asymptotic expansion in $M$ as

$$
Z\left(k, M, \mathcal{C}_{\phi}\right) \simeq \sum_{n=0}^{\infty} Z_{n}, \quad Z_{n}=\frac{1}{n !} \int_{-\infty}^{\infty} d \phi\left(M \phi^{3}\right)^{n} e^{-k \phi^{2}} .
$$


Each $Z_{n}$ gives the partition function of a statistical system on random networks of $n$ trivalent vertices [47, 48]. In this paper, we do not generally assume the positivity of $k$ or the integration contour like (5.3), since our interest is in the solutions to the constraint equations rather than such statistical systems. As we will see shortly, the necessary properties are the well-definedness of the integral (5.1) in the space of $M$ except for possible singularities, and the validity of the partial integrations over $\phi_{a}$ 's which will be performed in the rest of this paper.

Firstly, one can show that the partition function (5.4) satisfies the momentum constraints (2.15), because

$$
\begin{aligned}
\hat{\mathcal{J}}_{[a b]} Z\left(k, M, \mathcal{C}_{\phi}\right) & \propto\left(M_{a c d} D_{b c d}^{M}-M_{b c d} D_{a c d}^{M}\right) Z\left(k, M, \mathcal{C}_{\phi}\right) \\
& =\int_{\mathcal{C}_{\phi}} d \phi\left(M_{a c d} \phi_{b} \phi_{c} \phi_{d}-M_{b c d} \phi_{a} \phi_{c} \phi_{d}\right) e^{-k \phi^{2}+M \phi^{3}} \\
& =\frac{1}{3} \int_{\mathcal{C}_{\phi}} d \phi e^{-k \phi^{2}}\left(\phi_{b} D_{a}^{\phi}-\phi_{a} D_{b}^{\phi}\right) e^{M \phi^{3}} \\
& =-\frac{1}{3} \int_{\mathcal{C}_{\phi}} d \phi e^{M \phi^{3}}\left[D_{a}^{\phi}\left(\phi_{b} e^{-k \phi^{2}}\right)-D_{b}^{\phi}\left(\phi_{a} e^{-k \phi^{2}}\right)\right]=0,
\end{aligned}
$$

where we have used a short-hand notation,

$$
D_{a}^{\phi} \equiv \frac{\partial}{\partial \phi_{a}},
$$

and have assumed the validity of the partial integrations over $\phi_{a}$ 's. This is justified, if $\mathcal{C}_{\phi}$ is a closed curve, or if the integrand damps rapidly enough in the case that $\mathcal{C}_{\phi}$ extends to infinity. In the rest of this paper, without being explicitly mentioned, we simply assume that all the partial integrations (not only over $\phi_{a}$ 's but also over some other variables) performed in due course be valid.

Next, let us consider the Hamiltonian constraints. From (2.14), the corresponding partial differential equations for $\lambda=0$ in the $M$-representation are given by

$$
\left(M_{b d e} D_{a b c}^{M} D_{c d e}^{M}+\lambda_{H} D_{a b b}^{M}\right) \psi(M)=0 .
$$

As for the first term, we obtain

$$
\begin{aligned}
M_{b d e} D_{a b c}^{M} D_{c d e}^{M} Z\left(k, M, \mathcal{C}_{\phi}\right) & =\int_{\mathcal{C}_{\phi}} d \phi M_{b d e} \phi_{a} \phi_{b} \phi_{c} \phi_{c} \phi_{d} \phi_{e} e^{-k \phi^{2}+M \phi^{3}} \\
& =\int_{\mathcal{C}_{\phi}} d \phi \phi_{a} \phi^{2} M_{b d e} \phi_{b} \phi_{d} \phi_{e} e^{-k \phi^{2}+M \phi^{3}} \\
& =\frac{1}{3} \int_{\mathcal{C}_{\phi}} d \phi \phi_{a} \phi^{2} e^{-k \phi^{2}} \phi_{b} D_{b}^{\phi} e^{M \phi^{3}} \\
& =-\frac{1}{3} \int_{\mathcal{C}_{\phi}} d \phi e^{M \phi^{3}} D_{b}^{\phi}\left(\phi_{a} \phi_{b} \phi^{2} e^{-k \phi^{2}}\right) \\
& =-\int_{\mathcal{C}_{\phi}} d \phi \phi_{a} \phi^{2}\left(1+\frac{N}{3}-\frac{2 k}{3} \phi^{2}\right) e^{-k \phi^{2}+M \phi^{3}} \\
& =-\int_{\mathcal{C}_{\phi}} d \phi \phi_{a} \phi^{2}\left(1+\frac{N}{3}+\frac{2}{3} k \frac{\partial}{\partial k}\right) e^{-k \phi^{2}+M \phi^{3}} .
\end{aligned}
$$


As for the second term of (5.7), we obtain

$$
D_{a b b}^{M} Z\left(k, M, \mathcal{C}_{\phi}\right)=\int_{\mathcal{C}_{\phi}} d \phi \phi_{a} \phi^{2} e^{-k \phi^{2}+M \phi^{3}}
$$

Because of the derivative term with respect to $k$ in the last line of (5.8), the partition function itself does not satisfy the Hamiltonian constraints. This can be remedied by introducing a function $g(k)$ and the integration over $k$. To see this, let us define

$$
\psi_{\mathcal{C}_{k, \phi}}(M)=\int_{\mathcal{C}_{k}} d k g(k) Z\left(k, M, \mathcal{C}_{\phi}\right)=\int_{\mathcal{C}_{k, \phi}} d k d \phi g(k) e^{-k \phi^{2}+M \phi^{3}}
$$

Then

$$
\begin{aligned}
\left(M_{b d e} D_{a b c}^{M} D_{c d e}^{M}+\lambda_{H} D_{a b b}^{M}\right) \psi_{\mathcal{C}_{k, \phi}}(M) \\
\quad=\int_{\mathcal{C}_{k, \phi}} d k d \phi g(k) \phi_{a} \phi^{2}\left[-\left(1+\frac{N}{3}+\frac{2}{3} k \frac{\partial}{\partial k}\right)+\lambda_{H}\right] e^{-k \phi^{2}+M \phi^{3}} \\
=\frac{2}{3} \int_{\mathcal{C}_{k, \phi}} d k d \phi \phi_{a} \phi^{2}\left(k g^{\prime}(k)+\alpha_{N} g(k)\right) e^{-k \phi^{2}+M \phi^{3}}
\end{aligned}
$$

where we have performed a partial integration over $k$ under the assumption of its validity, and

$$
\alpha_{N}=\frac{3 \lambda_{H}-N-1}{2}=\frac{N^{2}+N+2}{8} .
$$

Therefore the Hamiltonian constraints are satisfied, if we take

$$
g(k)=k^{-\alpha_{N}} .
$$

By considering various choices of the integration domain, $\mathcal{C}_{k, \phi}$, one can obtain a number of independent solutions.

Physics behind the present solution would be given as follows. As discussed in the previous paper [47], a flow generated by a scale-free $O(N)$-invariant linear combination of the Hamiltonian constraints of the canonical tensor model could be interpreted as a renormalization group flow of statistical systems on random networks. ${ }^{8}$ A renormalization group procedure, if existed, on random networks would change the number of vertices of networks, that is a natural analog of a block spin transformation on a regular lattice. Therefore, since the parameter $k$ controls the relative weights among networks of $n$ trivalent vertices as $\propto k^{-\frac{3 n}{2}}$, a renormalization group procedure should generate a flow in $k$. This would be in accordance with the appearance of a derivative term of $k$ in the last line of (5.8), and it must be compensated by introducing $g(k)$ to make an invariant under the flow.

\footnotetext{
${ }^{8}$ Strictly speaking, this was discussed only for $N=2$ corresponding to the Ising model.
} 
Here let us check the type of the solution $\psi_{\mathcal{C}_{k, \phi}}$. We obtain

$$
\begin{aligned}
M_{a c d} D_{b c d}^{M} \psi_{\mathcal{C}_{k, \phi}}(M) & =\int_{\mathcal{C}_{k, \phi}} d k d \phi g(k) M_{a c d} \phi_{b} \phi_{c} \phi_{d} e^{-k \phi^{2}+M \phi^{3}} \\
& =\frac{1}{3} \int_{\mathcal{C}_{k, \phi}} d k d \phi g(k) e^{-k \phi^{2}} \phi_{b} D_{a}^{\phi} e^{M \phi^{3}} \\
& =-\frac{1}{3} \int_{\mathcal{C}_{k, \phi}} d k d \phi g(k) e^{M \phi^{3}} D_{a}^{\phi}\left(\phi_{b} e^{-k \phi^{2}}\right) \\
& =-\frac{1}{3} \int_{\mathcal{C}_{k, \phi}} d k d \phi g(k)\left(\delta_{a b}-2 k \phi_{a} \phi_{b}\right) e^{-k \phi^{2}+M \phi^{3}} .
\end{aligned}
$$

Here the second term in the last line cannot be concluded to be proportional to $\delta_{a b}$, and therefore $\hat{\mathcal{J}}_{(a b)} \psi_{\mathcal{C}_{k, \phi}}(M) \neq 0$ (See $(2.24)$ for $\hat{\mathcal{J}}_{(a b)}$ ) is expected in general. This means that $\psi_{\mathcal{C}_{k, \phi}}$ is a dynamical solution in general. However, there exists a delicate issue for $N=1,2$ as follows. By performing similar computations as (5.15), we obtain

$$
\left(M_{a b c} D_{a b c}^{M}+\lambda_{H}-1\right) \psi_{\mathcal{C}_{k, \phi}}(M)=0 .
$$

Then one finds that, for $N=1,2, \psi_{\mathcal{C}_{k, \phi}}(M)$ satisfies the necessary condition (2.29) to be kinematical. For $N=1,2$, we cannot ignore the possibility that $\psi_{\mathcal{C}_{k, \phi}}(M)$ may become kinematical. For $N \geq 3$, since the necessary condition is violated, the solution is definitely dynamical.

Finally, we would like to comment on the partition function (5.1) from a mathematical view point. Let us start with a generalization of the Airy function:

$$
\operatorname{Ai}\left[j, M, \mathcal{C}_{\phi}\right]=\int_{\mathcal{C}_{\phi}} d \phi e^{-j_{a} \phi_{a}+M_{a b c} \phi_{a} \phi_{b} \phi_{c}}
$$

where $j$ is a vector. (5.17) satisfies the following generalization of Airy's differential equation:

$$
\left(3 M_{a b c} \frac{\partial}{\partial j_{b}} \frac{\partial}{\partial j_{c}}-j_{a}\right) \operatorname{Ai}\left[j, M, \mathcal{C}_{\phi}\right]=0,
$$

which can be shown by partial integrations over $\phi$. The generalized Airy function can be related to the partition function (5.1) in the following manner. Firstly, one can remove the linear term in the exponent in (5.17) by a shift,

$$
\phi_{a} \rightarrow \phi_{a}+w_{a}
$$

where $w$ is a vector satisfying

$$
j_{a}-3 M_{a b c} w_{b} w_{c}=0
$$

Then there appears a quadratic term in $\phi, 3 M_{a b c} w_{c} \phi_{a} \phi_{b}$, as well as a constant term, $-j_{a} w_{a}+M_{a b c} w_{a} w_{b} w_{c}$. The quadratic term can be diagonalized and normalized by a linear transformation,

$$
\phi_{a} \rightarrow \phi_{b} R_{b a}
$$


with a complex matrix $R$, if the quadratic term is not singular. Then, (5.17) can be transformed to a form,

$$
\operatorname{Ai}\left[j, M, \mathcal{C}_{\phi}\right]=|R| e^{-j w+M w^{3}} \int_{\mathcal{C}_{\phi}^{\prime}} d \phi e^{-k \phi^{2}+M^{\prime} \phi^{3}}=|R| e^{-j w+M w^{3}} Z\left(k, M^{\prime}, \mathcal{C}_{\phi}^{\prime}\right),
$$

where

$$
M_{a b c}^{\prime}=R_{a a^{\prime}} R_{b b^{\prime}} R_{c c^{\prime}} M_{a^{\prime} b^{\prime} c^{\prime}}
$$

$k$ is a number, and $|R|$ denotes the determinant of $R$. Therefore the mathematical properties of the generalized Airy functions can be related to those of the partition function. This observation would be useful in future study.

\section{Simpler solutions}

The solution found in section 5 has a direct connection with statistical systems on random networks, and would therefore be interesting from physical viewpoints. On the other hand, if we set aside the physical interpretation, the solution (5.10) would be simplified by replacing $\int d k g(k) e^{-k \phi^{2}}$ with a function of $\phi^{2}$. Thus, in this section, let us assume a form,

$$
\psi_{\mathcal{C}_{\phi}, h}(M)=\int_{\mathcal{C}_{\phi}} d \phi f\left(\phi^{2}\right) h\left(M \phi^{3}\right),
$$

where $f$ and $h$ are functions of the shown arguments, and solve the constraints. $f$ will be determined shortly.

Let us first check the momentum constraints. We obtain

$$
\begin{aligned}
\left(M_{a c d} D_{b c d}^{M}-M_{b c d} D_{a c d}^{M}\right) \psi_{\mathcal{C}_{\phi}, h}(M) & =\int_{\mathcal{C}_{\phi}} d \phi f\left(\phi^{2}\right)\left(M_{a c d} \phi_{b} \phi_{c} \phi_{d}-M_{b c d} \phi_{a} \phi_{c} \phi_{d}\right) h^{\prime}\left(M \phi^{3}\right) \\
& =\frac{1}{3} \int_{\mathcal{C}_{\phi}} d \phi f\left(\phi^{2}\right)\left(\phi_{b} D_{a}^{\phi}-\phi_{a} D_{b}^{\phi}\right) h\left(M \phi^{3}\right) \\
& =-\frac{1}{3} \int_{\mathcal{C}_{\phi}} d \phi h\left(M \phi^{3}\right)\left(D_{a}^{\phi}\left(\phi_{b} f\left(\phi^{2}\right)\right)-D_{b}^{\phi}\left(\phi_{a} f\left(\phi^{2}\right)\right)\right) \\
& =0 .
\end{aligned}
$$

As for the Hamiltonian constraints, we first obtain

$$
\begin{aligned}
M_{b d e} D_{a b c}^{M} D_{c d e}^{M} \int_{\mathcal{C}_{\phi}} d \phi f\left(\phi^{2}\right) h\left(M \phi^{3}\right) & =\int_{\mathcal{C}_{\phi}} d \phi M_{b d e} \phi_{a} \phi_{b} \phi_{c} \phi_{c} \phi_{d} \phi_{e} f\left(\phi^{2}\right) h^{\prime \prime}\left(M \phi^{3}\right) \\
& =\int_{\mathcal{C}_{\phi}} d \phi f\left(\phi^{2}\right) \phi_{a} \phi_{b} \phi^{2} \frac{1}{3} D_{b}^{\phi} h^{\prime}\left(M \phi^{3}\right) \\
& =-\frac{1}{3} \int_{\mathcal{C}_{\phi}} d \phi h^{\prime}\left(M \phi^{3}\right) D_{b}^{\phi}\left(f\left(\phi^{2}\right) \phi_{a} \phi_{b} \phi^{2}\right) \\
& =-\frac{1}{3} \int_{\mathcal{C}_{\phi}} d \phi \phi_{a}\left((N+1) \tilde{f}\left(\phi^{2}\right)+2 \phi^{2} \tilde{f}^{\prime}\left(\phi^{2}\right)\right) h^{\prime}\left(M \phi^{3}\right),
\end{aligned}
$$


where $\tilde{f}(x)=x f(x)$. Similarly,

$$
\begin{aligned}
D_{a b b}^{M} \int_{\mathcal{C}_{\phi}} d \phi f\left(\phi^{2}\right) h\left(M \phi^{3}\right) & =\int_{\mathcal{C}_{\phi}} d \phi f\left(\phi^{2}\right) \phi_{a} \phi_{b} \phi_{b} h^{\prime}\left(M \phi^{3}\right), \\
& =\int_{\mathcal{C}_{\phi}} d \phi \phi_{a} \tilde{f}\left(\phi^{2}\right) h^{\prime}\left(M \phi^{3}\right) .
\end{aligned}
$$

Therefore,

$$
\left(M_{b d e} D_{a b c}^{M} D_{c d e}^{M}+\lambda_{H} D_{a b b}^{M}\right) \psi_{h, \mathcal{C}_{\phi}}(M)=\frac{2}{3} \int_{\mathcal{C}_{\phi}} d \phi \phi_{a}\left(\alpha_{N} \tilde{f}\left(\phi^{2}\right)-\phi^{2} \tilde{f}^{\prime}\left(\phi^{2}\right)\right) h^{\prime}\left(M \phi^{3}\right)
$$

where $\alpha_{N}$ was defined in (5.13). Thus, by putting

$$
\tilde{f}(x)=x^{\alpha_{N}},
$$

a solution to the constraint equations can be obtained as

$$
\psi_{\mathcal{C}_{\phi}, h}(M)=\int_{\mathcal{C}_{\phi}} d \phi\left(\phi^{2}\right)^{\beta_{N}} h\left(M \phi^{3}\right)
$$

where $\beta_{N}=\alpha_{N}-1=\frac{(N-2)(N+3)}{8}$. One would be able to obtain a number of independent solutions by considering various $\mathcal{C}_{\phi}$ and $h$.

One can obtain another kind of solutions by determining $h$ instead of $f$ in (6.1). In this case, we have

$$
\begin{aligned}
\left(M_{c d e} D_{a b c}^{M} D_{b d e}^{M}+\right. & \left.\lambda_{H} D_{a b b}^{M}\right) \int_{\mathcal{C}_{\phi}} d \phi f\left(\phi^{2}\right) h\left(M \phi^{3}\right) \\
= & \int_{\mathcal{C}_{\phi}} d \phi \phi_{a} \phi^{2} f\left(\phi^{2}\right)\left(M \phi^{3} h^{\prime \prime}\left(M \phi^{3}\right)+\lambda_{H} h^{\prime}\left(M \phi^{3}\right)\right) .
\end{aligned}
$$

This vanishes, if

$$
h(x)=x^{-\gamma_{N}}+\text { const. }
$$

where $\gamma_{N}=\lambda_{H}-1=\frac{(N-1)(N+6)}{12}$. The constant term is irrelevant, since it does not produce any dependence on $M$. Thus a non-tivial solution to the constraints is given by

$$
\psi_{\mathcal{C}_{\phi}, f}(M)=\int_{\mathcal{C}_{\phi}} d \phi\left(M \phi^{3}\right)^{-\gamma_{N}} f\left(\phi^{2}\right) .
$$

Various choices of $\mathcal{C}_{\phi}, f$ will provide a number of independent solutions.

For instance, by considering $f(x)=e^{-x}$ in (6.10), we obtain a similar expression as $Z_{n}$ in (5.4). Therefore, in the large- $N$ limit, one would be able to employ a similar saddle-point method used in the analysis of the thermodynamic limit of statistical systems on random networks in $[47,48]$. However, there exist at least the following major differences from the statistical systems: the signature of the exponent is opposite, and one can more freely take the integration domain, $\mathcal{C}_{\phi}$. Therefore, the dynamics would be much different. We leave this interesting aspect for future analysis. 
From (6.7) and (6.10), it is obvious that

$$
\psi_{\mathcal{C}_{\phi}}(M)=\int_{\mathcal{C}_{\phi}} d \phi\left(\phi^{2}\right)^{\beta_{N}}\left(M \phi^{3}\right)^{-\gamma_{N}}
$$

is a solution. In this case, the dimension of $\phi$ is canceled in the integration, since $N+$ $2 \beta_{N}-3 \gamma_{N}=0$. Therefore, it should be the canonical form of the solution in the case that $\mathcal{C}_{\phi}$ is a finite domain.

Let us compute the scaling dimension of the above solutions. We easily obtain

$$
M_{a b c} D_{a b c}^{M} \psi(M)=-\gamma_{N} \psi(M)
$$

for all the solutions above, and this coincides with (5.16). Then the solutions in this section are dynamical except for the delicate case $N=1,2$ as discussed in section 5 .

\section{$7 \quad$ Explicit examples}

In this section, we will check the solutions for the simple cases with $N=1,2$.

\section{1 $\quad N=1$}

For the $N=1$ case, taking $h(x)=1$ in (6.7) gives

$$
\psi=\oint \frac{d \phi}{\phi}=2 \pi i
$$

Here the contour is assumed to be a closed path surrounding the origin. This is actually the trivial solution to the constraint equation for $N=1$,

$$
D^{M} M D^{M} \psi=0 .
$$

To obtain a non-trivial solution, we consider (6.7) with $h(x)=\log (x)$ :

$$
\psi_{\log }(M)=\oint \frac{d \phi}{\phi} \log \left(M \phi^{3}\right)=2 \pi i \log M+\text { const. },
$$

which is indeed the other independent solution to (7.2). Here the multi-valuedness of the logarithmic function in the integrand is not problematic: the first derivative, $h^{\prime}(x)=\frac{1}{x}$, is a single-valued function, and one can safely justify the partial integration performed in (6.3). Since $M D^{M} \psi_{\log } \neq 0$, this is a dynamical solution.

\section{$7.2 \quad N=2$}

For the $N=2$ case, (6.11) gives

$$
\psi(M)=\int_{\mathcal{C}_{\phi}} d \phi_{1} d \phi_{2} \frac{1}{\left(M_{a b c} \phi_{a} \phi_{b} \phi_{c}\right)^{\frac{2}{3}}} .
$$

Let us check if one can really choose the contour, $\mathcal{C}_{\phi}$, so that (7.4) becomes a non-vanishing meaningful solution. To begin, when choosing the gauge,

$$
M_{111}=1, \quad M_{112}=0, \quad M_{122}=x_{1}, \quad M_{222}=x_{2},
$$




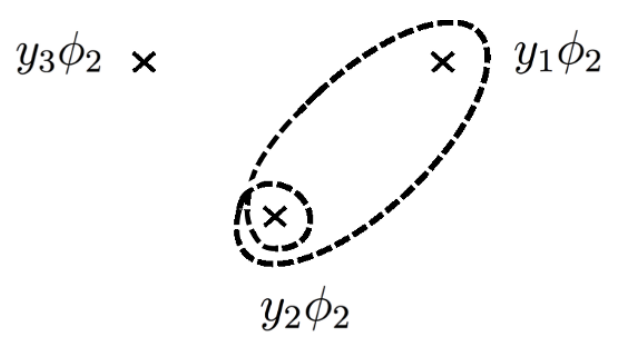

Figure 1. A proper contour, $\mathcal{C}_{\phi_{1}}$ : a closed dashed line winding two times around $\phi_{1}=y_{2} \phi_{2}$ and one time around $\phi_{1}=y_{1} \phi_{2}$.

one finds

$$
\begin{aligned}
M_{a b c} \phi_{a} \phi_{b} \phi_{c} & =\phi_{1}{ }^{3}+3 x_{1} \phi_{1} \phi_{2}{ }^{2}+x_{2} \phi_{2}{ }^{3} \\
& =\left(\phi_{1}-y_{1} \phi_{2}\right)\left(\phi_{1}-y_{2} \phi_{2}\right)\left(\phi_{1}-y_{3} \phi_{2}\right),
\end{aligned}
$$

where $y_{i}$ 's are the three solutions to $y^{3}+3 x_{1} y+x_{2}=0$. We then implement the $\phi_{1}$ integration by choosing the contour, $\mathcal{C}_{\phi_{1}}$, in such a way as to enclose $\phi_{1}=y_{2} \phi_{2}$ two times and $\phi_{1}=y_{1} \phi_{2}$ one time (see figure 1 ). We take such a contour to make it consistent with the multi-valuedness of the integrand caused by the fractional power. This choice of contour is in fact democratic: every contour enclosing $\phi_{1}=y_{i} \phi_{2}(i=1,2,3)$ two times and $\phi_{1}=y_{j} \phi_{2}(j \neq i)$ one time yield the same result up to a sign. After the $\phi_{1}$-integration, (7.4) can be shown to be

$$
\text { const. } \int_{\mathcal{C}_{\phi_{2}}} d \phi_{2} \frac{1}{\phi_{2}\left[\left(y_{1}-y_{2}\right)\left(y_{2}-y_{3}\right)\left(y_{3}-y_{1}\right)\right]^{\frac{1}{3}}} .
$$

Choosing $\mathcal{C}_{\phi_{2}}$ to enclose $\phi_{2}=0$, one finally finds

$$
\psi(M) \propto \frac{1}{\left[\left(y_{1}-y_{2}\right)\left(y_{2}-y_{3}\right)\left(y_{3}-y_{1}\right)\right]^{\frac{1}{3}}} \propto \frac{1}{\left(4 x_{1}^{3}+x_{2}^{2}\right)^{\frac{1}{6}}} \propto A(M)^{-\frac{1}{6}},
$$

where the last expression is valid in the whole space with $A(M)$ defined in (3.13). This can be shown to be a kinematical solution, similarly to (3.16).

\section{Solutions in $\boldsymbol{P}$-representation without a cosmological constant}

The method in section 6 can also be used to solve the constraint equations in the $P$ representation. Let us start with an assumption,

$$
Q_{\mathcal{C}_{\phi}, h}(P)=\int_{\mathcal{C}_{\phi}} d \phi f\left(\phi^{2}\right) h\left(P \phi^{3}\right),
$$

where $f$ will be determined shortly.

The momentum constraints can be shown to be satisfied by the same reasoning as in section 6. For $\lambda=0$ in (2.14), the Hamiltonian constraints are given by

$$
\left(P_{a b c} P_{b d e} D_{c d e}^{P}+\lambda_{H} P_{a b b}\right) \psi(P)=0 .
$$


As for the first term,

$$
\begin{aligned}
P_{a b c} P_{b d e} D_{c d e}^{P} Q_{\mathcal{C}_{\phi}, h}(P) & =\int_{\mathcal{C}_{\phi}} d \phi P_{a b c} P_{b d e} \phi_{c} \phi_{d} \phi_{e} f\left(\phi^{2}\right) h^{\prime}\left(P \phi^{3}\right) \\
& =\frac{1}{3} \int_{\mathcal{C}_{\phi}} d \phi P_{a b c} \phi_{c} f\left(\phi^{2}\right) D_{b}^{\phi} h\left(P \phi^{3}\right) \\
& =-\frac{1}{3} \int_{\mathcal{C}_{\phi}} d \phi D_{b}^{\phi}\left(P_{a b c} \phi_{c} f\left(\phi^{2}\right)\right) h\left(P \phi^{3}\right) \\
& =-\frac{1}{3} \int_{\mathcal{C}_{\phi}} d \phi\left(P_{a b b} f\left(\phi^{2}\right)+2 P_{a b c} \phi_{b} \phi_{c} f^{\prime}\left(\phi^{2}\right)\right) h\left(P \phi^{3}\right) .
\end{aligned}
$$

In the last line of (8.3), the second term seems to be problematic for the constraint equations to be satisfied. So, we set $f(x)=1$ to discard it. Then, from (8.3), we obtain that

$$
Q_{\mathcal{C}_{\phi}, h}(P)=\int_{\mathcal{C}_{\phi}} d \phi h\left(P \phi^{3}\right)
$$

satisfies

$$
\left(P_{a b c} P_{b d e} D_{c d e}^{P}+\frac{1}{3} P_{a b b}\right) Q_{\mathcal{C}_{\phi}, h}(P)=0 .
$$

The only difference of (8.2) from (8.5) is the numerical coefficient of the second term, and it is obvious that

$$
\psi_{\mathcal{C}_{\phi}, h}(P)=Q_{\mathcal{C}_{\phi}, h}(P)^{3 \lambda_{H}}
$$

satisfies the constraint equations (8.2).

The scaling dimension can be computed as

$$
\begin{aligned}
P_{a b c} D_{a b c}^{P} Q_{\mathcal{C}_{\phi}, h}(P) & =\int_{\mathcal{C}_{\phi}} d \phi\left(P \phi^{3}\right) h^{\prime}\left(P \phi^{3}\right) \\
& =\frac{1}{3} \int_{\mathcal{C}_{\phi}} d \phi \phi_{a} D_{a}^{\phi} h\left(P \phi^{3}\right) \\
& =-\frac{N}{3} Q_{\mathcal{C}_{\phi}, h}(P) .
\end{aligned}
$$

Thus,

$$
P_{a b c} D_{a b c}^{P} \psi_{\mathcal{C}_{\phi}, h}(P)=-N \lambda_{H} \psi_{\mathcal{C}_{\phi}, h}(P)
$$

which agrees with the necessary condition (2.28) for a kinematical solution. The scaling dimension will become obvious, if we take $h(x)=x^{-\frac{N}{3}}$ as

$$
Q_{\mathcal{C}_{\phi}}(P)=\int_{\mathcal{C}_{\phi}} d \phi\left(P \phi^{3}\right)^{-\frac{N}{3}}
$$

This should be a canonical expression when $\mathcal{C}_{\phi}$ is a finite domain, since the dimension of $\phi$ is canceled in the integration. 
Finally, let us check the type of the solution (8.6). By partial integrations, one obtains

$$
\begin{aligned}
P_{a c d} D_{b c d}^{P} Q_{\mathcal{C}_{\phi}, h}(P) & =\int_{\mathcal{C}_{\phi}} d \phi P_{a c d} \phi_{b} \phi_{c} \phi_{d} h^{\prime}\left(P \phi^{3}\right) \\
& =\frac{1}{3} \int_{\mathcal{C}_{\phi}} d \phi \phi_{b} D_{a}^{\phi} h\left(P \phi^{3}\right) \\
& =-\frac{1}{3} \delta_{a b} Q_{\mathcal{C}_{\phi}, h}(P) .
\end{aligned}
$$

This leads to $\hat{\mathcal{J}}_{(a b)} \psi_{\mathcal{C}_{\phi}, h}(P)=0$, namely, $\psi_{\mathcal{C}_{\phi}, h}(P)$ is a kinematical solution, and might not be so interesting by itself. However, it will be useful as a component to generate a variety of dynamical solutions in the following sense. Let us suppose that we have two solutions, say $\psi_{1}(P), \psi_{2}(P)$, which both satisfy the constraint equations (8.2). Then, it is obvious that the product, $\psi_{1}(P)^{\tau} \psi_{2}(P)^{1-\tau}$, with an arbitrary number $\tau$ satisfies (8.2). Namely, one can construct one parameter family of solutions to the constraint equations from two independent solutions. This essentially comes from the fact that the Hamiltonian constraints are first-order differential operators in the $P$-representation. Now, a number of dynamical solutions in the $P$-representation can be obtained through the Fourier transform of the solutions in the $M$-representation in section 5 and section 6 . Then, the above products of these solutions and $\psi_{\mathcal{C}_{\phi}, h}(P)$ will give families of dynamical solutions.

\section{Solutions with integration over matrix and tensor variables}

The construction of the solutions in section 8 can be generalized to that with integration over matrix and tensor variables. The first two solutions below are kinematical, while the final one is dynamical.

Let us consider

$$
Q_{\mathcal{C}_{K, \phi}, h, g}(P)=\int_{\mathcal{C}_{K, \phi}} d K d \phi h(P K \phi) g\left(P \phi^{3}\right)
$$

where we have introduced an integration over a symmetric matrix $K$, in addition to the integration over $\phi$ as before, $h, g$ are functions, and

$$
P K \phi \equiv P_{a b c} K_{a b} \phi_{c}
$$

By performing partial integrations as in the previous sections, one can show that

$$
\begin{aligned}
P_{a c d} & D_{b c d}^{P} Q_{\mathcal{C}_{K, \phi}, h, g}(P) \\
& =\int_{\mathcal{C}_{K, \phi}} d K d \phi P_{a c d}\left\{\frac{1}{3}\left(2 K_{b c} \phi_{d}+K_{c d} \phi_{b}\right) h^{\prime}(P K \phi) g\left(P \phi^{3}\right)+\phi_{b} \phi_{c} \phi_{d} h(P K \phi) g^{\prime}\left(P \phi^{3}\right)\right\} \\
& =\frac{1}{3} \int_{\mathcal{C}_{K, \phi}} d K d \phi\left(2 K_{b c} D_{c a}^{K}+\phi_{b} D_{a}^{\phi}\right) h(P K \phi) g\left(P \phi^{3}\right) \\
& =-\frac{N+2}{3} \delta_{a b} Q_{\mathcal{C}_{K, \phi}, h, g}(P),
\end{aligned}
$$


where $D^{K}$ denotes partial derivatives with respect to $K$ as

$$
D_{a b}^{K} K_{c d}=\frac{1}{2}\left(\delta_{a c} \delta_{b d}+\delta_{a d} \delta_{b c}\right) .
$$

Then,

$$
\psi_{\mathcal{C}_{K, \phi}, h, g}(P)=Q_{\mathcal{C}_{K, \phi}, h, g}(P)^{\frac{3 \lambda_{H}}{N+2}}
$$

satisfies the constraint equations (8.2). (9.3) also shows that $\psi_{\mathcal{C}_{K, \phi}, h, g}(P)$ is a kinematical solution.

A solution with integration over a tensor variable starts with considering

$$
Q_{\mathcal{C}_{T}, h}(P)=\int_{\mathcal{C}_{T}} d T h(P T),
$$

where $T$ is a symmetric tensor with three indices, and

$$
P T \equiv P_{a b c} T_{a b c} .
$$

By partial integrations as before, one can show that

$$
P_{a c d} D_{b c d}^{P} Q_{\mathcal{C}_{T}, h}(P)=-\frac{(N+2)(N+1)}{6} \delta_{a b} Q_{\mathcal{C}_{T}, h}(P) .
$$

Then

$$
\psi_{\mathcal{C}_{T}, h}(P)=Q_{\mathcal{C}_{T}, h}(P)^{\frac{6 \lambda_{H}}{(N+1)(N+2)}}
$$

can be shown to satisfy the constraint equations (8.2). (9.8) also shows that $\psi_{\mathcal{C}_{T}, h}(P)$ is a kinematical solution.

So far, in this and previous sections, we have only constructed kinematical solutions in the $P$-representation. A dynamical solution can be constructed by starting with the following integration over a symmetric matrix $K$,

$$
Q_{\mathcal{C}_{K}, h}(P)=\int_{\mathcal{C}_{K}} d K h(K P P K)
$$

where

$$
K P P K=K_{a b} P_{a b c} P_{c d e} K_{d e} .
$$

As for the first term of the Hamiltonian constraints,

$$
\begin{aligned}
P_{a b c} P_{b d e} D_{c d e}^{P} Q_{\mathcal{C}_{K}, h}(P) & =2 \int_{\mathcal{C}_{K}} d K P_{a b c} P_{b d e} K_{\underline{c d}}(P K)_{\underline{e}} h^{\prime}(K P P K) \\
& =\frac{2}{3} \int_{\mathcal{C}_{K}} d K\left(P_{a b c}(P K)_{b}(P K)_{c}+2 P_{a b c} P_{b d e} K_{c d}(P K)_{e}\right) h^{\prime}(K P P K) \\
& =\frac{1}{3} \int_{\mathcal{C}_{K}} d K\left((P K)_{b} D_{a b}^{K}+2 P_{a b c} K_{c d} D_{b d}^{K}\right) h(K P P K) \\
& =-\frac{1}{3} \int_{\mathcal{C}_{K}} d K\left(\left(D_{a b}^{K}(P K)_{b}\right)+2 P_{a b c}\left(D_{b d}^{K} K_{c d}\right)\right) h(K P P K) \\
& =-\frac{1}{3} \int_{\mathcal{C}_{K}} d K\left(P_{a b b}+(N+1) P_{a b b}\right) h(K P P K) \\
& =-\frac{N+2}{3} P_{a b b} Q_{\mathcal{C}_{K}, h}(P),
\end{aligned}
$$


where the underlined indices are supposed to take the average over their permutations, and

$$
(P K)_{a} \equiv P_{a b c} K_{b c}
$$

In a similar way, the scaling dimension can be obtained as

$$
P_{a b c} D_{a b c}^{P} Q_{\mathcal{C}_{K}, h}(P)=-\frac{N(N+1)}{2} Q_{\mathcal{C}_{K}, h}(P) .
$$

These results show that

$$
\psi_{\mathcal{C}_{K}, h}(P)=Q_{\mathcal{C}_{K}, h}(P)^{\frac{3 \lambda_{H}}{N+2}}
$$

is a solution to the constraint equations, and its scaling dimension is given by

$$
P_{a b c} D_{a b c}^{P} \psi_{\mathcal{C}_{K}, h}(P)=-\frac{3 N(N+1) \lambda_{H}}{2(N+2)} \psi_{\mathcal{C}_{K}, h}(P)
$$

By comparing with $(2.28), \psi_{\mathcal{C}_{K}, h}(P)$ is a dynamical solution for $N>1$.

It would be meaningful to see whether $\psi_{\mathcal{C}_{K}, h}(P)$ is different from the solutions obtained in section 5 and 6 . From (6.12), we obtain

$$
-P_{a b c} D_{a b c}^{P} \psi(P)=i \hat{P}_{a b c} \hat{M}_{a b c} \psi=D_{a b c}^{M} M_{a b c} \psi(M)=\left(\frac{N(N+1)(N+2)}{6}-\gamma_{N}\right) \psi(M)
$$

This differs from (9.16) except for $N=1,4$. Therefore, $\psi_{\mathcal{C}_{K}, h}(P)$ is a new solution at least for $N \neq 1,4$.

\section{Solutions in $P$-representation with a cosmological constant}

The canonical tensor model with variables satisfying the generalized hermiticity condition does not allow a cosmological constant term, because such a term violates the consistency of the first-class constraint algebra [40]. On the other hand, if we restrict the variables to be real symmetric as in this paper, the algebra remains consistent even after such a cosmological term is introduced as in section 2. The Hamiltonian constraint equations with a cosmological constant $\lambda$ in the $P$-representation are given by

$$
\left(P_{a b c} P_{b d e} D_{c d e}^{P}+\lambda_{H}^{N} P_{a b b}-\lambda D_{a b b}^{P}\right) \psi(P)=0,
$$

where the dependence of the normal ordering term on $N$ is explicitly written for later convenience. The interpretation of $\lambda$ as a cosmological constant has been validated by comparing the classical dynamics of the canonical tensor model with $N=1$ and the minisuperspace approximation of general relativity [46].

In this section, we will explicitly construct a few series of solutions to (10.1) by generalizing the solutions obtained in the previous sections. Remarkably, it will be observed that all these solutions for $N=m$ can actually be obtained from those of the constraint equations with no cosmological constant for $N=m+1$ by fixing the extra components of $P$. In section 11, we will prove a general theorem on this aspect. 
Let us start with (8.3),

$$
P_{a b c} P_{b d e} D_{c d e}^{P} Q_{\mathcal{C}_{\phi}, f, h}(P)+\frac{1}{3} \int_{\mathcal{C}_{\phi}} d \phi\left[P_{a b b} f\left(\phi^{2}\right)+2 P_{a b c} \phi_{b} \phi_{c} f^{\prime}\left(\phi^{2}\right)\right] h\left(P \phi^{3}\right)=0,
$$

where $Q_{\mathcal{C}_{\phi}, f, h}(P)$ is assumed to have the expression on the righthand side of (8.1). By performing a partial integration, the last term can be computed to be

$$
\begin{aligned}
\frac{2}{3} \int_{\mathcal{C}_{\phi}} d \phi P_{a b c} \phi_{b} \phi_{c} f^{\prime}\left(\phi^{2}\right) h\left(P \phi^{3}\right) & =\frac{2}{9} \int_{\mathcal{C}_{\phi}} d \phi f^{\prime}\left(\phi^{2}\right) D_{a}^{\phi} h^{I}\left(P \phi^{3}\right) \\
& =-\frac{4}{9} \int_{\mathcal{C}_{\phi}} d \phi \phi_{a} f^{\prime \prime}\left(\phi^{2}\right) h^{I}\left(P \phi^{3}\right),
\end{aligned}
$$

where $h^{I}(x)$ is a function satisfying

$$
\frac{d}{d x} h^{I}(x)=h(x)
$$

Now let us assume

$$
\begin{aligned}
h^{I}(x) & =\frac{1}{A} \exp [A x], \\
f^{\prime \prime}(x) & =B x f(x),
\end{aligned}
$$

with numerical constants, $A, B$. Then (10.3) can further be computed as

$$
\begin{aligned}
-\frac{4}{9} \int_{\mathcal{C}_{\phi}} d \phi \phi_{a} f^{\prime \prime}\left(\phi^{2}\right) h^{I}\left(P \phi^{3}\right) & =-\frac{4 B}{9 A} \int_{\mathcal{C}_{\phi}} d \phi \phi_{a} \phi^{2} f\left(\phi^{2}\right) h\left(P \phi^{3}\right) \\
& =-\frac{4 B}{9 A^{2}} D_{a b b}^{P} \int_{\mathcal{C}_{\phi}} d \phi f\left(\phi^{2}\right) h\left(P \phi^{3}\right) .
\end{aligned}
$$

This concludes

$$
\left[P_{a b c} P_{b d e} D_{c d e}^{P}+\frac{1}{3} P_{a b b}-\frac{4 B}{9 A^{2}} D_{a b b}^{P}\right] Q_{\mathcal{C}_{\phi}, f, h}(P)=0 .
$$

Thus, if we consider

$$
\psi_{\mathcal{C}_{\phi}, f, h}(P)=Q_{\mathcal{C}_{\phi}, f, h}(P)^{3 \lambda_{H}^{N}},
$$

we obtain

$$
\left[P_{a b c} P_{b d e} D_{c d e}^{P}+\lambda_{H}^{N} P_{a b b}-\frac{4 B}{9 A^{2}} D_{a b b}^{P}\right] \psi_{\mathcal{C}_{\phi}, f, h}(P)=0 .
$$

Namely, $\psi_{\mathcal{C}_{\phi}, f, h}(P)$ satisfies the constraint equations with a cosmological constant,

$$
\lambda=\frac{4 B}{9 A^{2}} .
$$

In fact, the solution to (10.6) is given by the Airy function,

$$
f(x)=\operatorname{Airy}\left[B^{\frac{1}{3}} x\right]=\int_{\mathcal{C}_{z}} d z \exp \left[-B^{\frac{1}{3}} x z+\frac{z^{3}}{3}\right] .
$$


Then, by putting $h(x)=\exp (A x)$ and (10.12) into the expression (8.1), one obtains an intriguing expression,

$$
\begin{aligned}
Q_{\mathcal{C}_{\phi}, f, h}(P) & =\int_{\mathcal{C}_{\phi, z}} d \phi d z \exp \left[A\left(P \phi^{3}\right)-B^{\frac{1}{3}} \phi^{2} z+\frac{z^{3}}{3}\right] \\
& =\text { const. } \int_{\mathcal{C}_{\phi, z}} d \phi d z \exp \left[P \phi^{3}-\phi^{2} z+\frac{A^{2}}{3 B} z^{3}\right] \\
& =\text { const. } \int_{\mathcal{C}_{\phi}} \prod_{a=1}^{N+1} d \phi_{a} \exp \left[\tilde{P} \phi^{3}\right]
\end{aligned}
$$

with

$$
\begin{aligned}
\tilde{P}_{a b c} & =P_{a b c}, \\
\tilde{P}_{a b N+1} & =-\frac{1}{3} \delta_{a b}, \\
\tilde{P}_{a N+1 N+1} & =0, \\
\tilde{P}_{N+1 N+1 N+1} & =\frac{4}{27 \lambda},
\end{aligned}
$$

where we have rescaled $\phi, z$, and have renamed $z=\phi_{N+1}$. Thus, $Q_{\mathcal{C}_{\phi}, f, h}(\tilde{P})$ has the form as (8.4), and therefore $\psi_{\mathcal{C}_{\phi}, f, h}(\tilde{P})=Q_{\mathcal{C}_{\phi}, f, h}(\tilde{P})^{3 \lambda_{H}^{N+1}}$ actually satisfies the constraint equations with no cosmological constant for the variables $\tilde{P}$, as same as the wave-function in (8.6). This shows that the cosmological constant can be absorbed into some of the dynamical variables by increasing $N$.

Below, we will show that a similar fact holds also for a wider class of solutions. Let us consider the solution, $Q_{\mathcal{C}_{\phi}, h}(P)$ in (8.4), and start with (8.5),

$$
P_{a b c} P_{b d e} D_{c d e}^{P} Q_{\mathcal{C}_{\phi}, h}(P)+\frac{1}{3} P_{a b b} Q_{\mathcal{C}_{\phi}, h}(P)=0,
$$

where $a, b, c=1,2, \cdots, N+1$. Let us divide the index set as

$$
a=\left\{\begin{array}{l}
i \in I_{N}=\{1,2, \ldots, N\} \\
z=N+1
\end{array},\right.
$$

and consider a subspace in which $P$ 's containing the index $z$ are fixed as

$$
\begin{aligned}
P_{z z z} & =A, \\
P_{z z i} & =0, \\
P_{z i j} & =B \delta_{i j},
\end{aligned}
$$

where $i, j \in I_{N}$. Below, $i, j, \ldots$ will be used for the elements in $I_{N}$, and $a, b, \ldots$ for both $I_{N}$ and $z$.

On the subspace (10.17), the second term of (10.15) for $a=i \in I_{N}$ becomes

$$
\frac{1}{3} P_{i b b} Q_{\mathcal{C}_{\phi}, h}(P)=\frac{1}{3} P_{i j j} Q_{\mathcal{C}_{\phi}, h}(P) .
$$


On the subspace, the first term of (10.15) for $a=i$ can be evaluated as

$$
\begin{aligned}
& P_{i b c} P_{b d e} D_{c d e}^{P} Q_{\mathcal{C}_{\phi}, h}(P) \\
& \quad=\int_{\mathcal{C}_{\phi}} d \phi P_{i b c} P_{b d e} \phi_{c} \phi_{d} \phi_{e} h^{\prime}\left(P \phi^{3}\right) \\
& \quad=\int_{\mathcal{C}_{\phi}} d \phi\left[P_{i j k} P_{j l m} \phi_{k} \phi_{l} \phi_{m}+3 B P_{i j k} \phi_{j} \phi_{k} \phi_{z}+\left(2 B^{2}+A B\right) \phi_{i}\left(\phi_{z}\right)^{2}+B^{2} \phi_{i} \phi_{j}^{2}\right] h^{\prime}\left(P \phi^{3}\right),
\end{aligned}
$$

where it is important to notice that (10.17) do not contain any conditions on the corresponding derivatives, $D_{z a b}^{P}$. The following two identities hold for (10.17):

$$
\begin{aligned}
& 0=\int_{\mathcal{C}_{\phi}} d \phi D_{i}^{\phi}\left(\phi_{z} h\left(P \phi^{3}\right)\right)=\int_{\mathcal{C}_{\phi}} d \phi\left[3 P_{i j k} \phi_{j} \phi_{k} \phi_{z}+6 B \phi_{i} \phi_{z}^{2}\right] h^{\prime}\left(P \phi^{3}\right), \\
& 0=\int_{\mathcal{C}_{\phi}} d \phi D_{z}^{\phi}\left(\phi_{i} h\left(P \phi^{3}\right)\right)=\int_{\mathcal{C}_{\phi}} d \phi\left[3 A \phi_{i}\left(\phi_{z}\right)^{2}+3 B \phi_{i} \phi_{j}^{2}\right] h^{\prime}\left(P \phi^{3}\right) .
\end{aligned}
$$

These identities can be used to delete the terms, $P_{i j k} \phi_{j} \phi_{k} \phi_{z}$ and $\phi_{i}\left(\phi_{z}\right)^{2}$ in (10.19), to obtain

$$
\begin{aligned}
P_{i b c} P_{b d e} D_{c d e}^{P} Q_{\mathcal{C}_{\phi}, h}(P) & =\int_{\mathcal{C}_{\phi}} d \phi\left[P_{i j k} P_{j l m} \phi_{k} \phi_{l} \phi_{m}+\frac{4 B^{3}}{A} \phi_{i} \phi_{j}^{2}\right] h^{\prime}\left(P \phi^{3}\right) \\
& =\left[P_{i j k} P_{j l m} D_{k l m}^{P}+\frac{4 B^{3}}{A} D_{i j j}^{P}\right] Q_{\mathcal{C}_{\phi}, h}(P)
\end{aligned}
$$

Thus the wave-function (8.6) satisfies the constraint equations with a cosmological constant,

$$
\lambda=-\frac{4 B^{3}}{A} .
$$

\section{A theorem for ignoring a cosmological constant}

In this section, as generalization of section 10, we will prove that a solution to the constraint equations with no cosmological constant for $N=m+1$ can always generate a solution to the constraint equations with a cosmological constant for $N=m$ by fixing the extra components of $P$ as in (10.17) with $A=B$.

Let us divide the index set as in (10.16), and assume that $i, j, \ldots$ denote the elements in $I_{N}$, while $a, b, \ldots$ both $I_{N}$ and $z$. Let us assume that a wave-function $\psi(P)$ satisfies the constraint equations with no cosmological constant and the momentum constraints as

$$
\begin{aligned}
{\left[P_{a b c} P_{b d e} D_{c d e}^{P}+\lambda_{H}^{N+1} P_{a b b}\right] \psi(P) } & =0, \\
{\left[P_{a c d} D_{b c d}^{P}-P_{b c d} D_{a c d}^{P}\right] \psi(P) } & =0,
\end{aligned}
$$

where the dependence of the normal ordering term on $N+1$ is explicitly written. Let us assume (10.17) for $P_{z a b}$, while $P_{i j k}$ are left arbitrary. Then, on the subspace, the second term in (11.1) for $a=i$ is given by

$$
P_{i b b}=P_{i j j}
$$


As for the first term in (11.1), by putting (10.17), we obtain

$$
\begin{aligned}
P_{i b c} P_{b d e} D_{c d e}^{P} \psi(P)= & {\left[P_{i j k} P_{j d e} D_{k d e}^{P}+P_{i j z} P_{j d e} D_{z d e}^{P}+P_{i z j} P_{z d e} D_{j d e}^{P}\right] \psi(P) } \\
= & {\left[P_{i j k} P_{j l m} D_{k l m}^{P}+2 P_{i j k} P_{j l z} D_{k l z}^{P}+P_{i j z} P_{j k l} D_{z k l}^{P}+2 P_{i j z} P_{j k z} D_{z k z}^{P}\right.} \\
& \left.\quad+P_{i z j} P_{z k l} D_{j k l}^{P}+P_{i z j} P_{z z z} D_{j z z}^{P}\right] \psi(P) \\
= & {\left[P_{i j k} P_{j l m} D_{k l m}^{P}+3 B P_{i j k} D_{j k z}^{P}+\left(2 B^{2}+A B\right) D_{i z z}^{P}+B^{2} D_{i j j}^{P}\right] \psi(P) . }
\end{aligned}
$$

On the other hand, by putting (10.17) into (11.2) for $a=i, b=z$, we obtain

$$
\begin{aligned}
0 & =\left[P_{i a b} D_{z a b}^{P}-P_{z a b} D_{i a b}^{P}\right] \psi(P) \\
& =\left[P_{i j k} D_{z j k}^{P}+2 P_{i j z} D_{z j z}^{P}-P_{z j k} D_{i j k}^{P}-P_{z z z} D_{i z z}^{P}\right] \psi(P) \\
& =\left[P_{i j k} D_{j k z}^{P}+(2 B-A) D_{i z z}^{P}-B D_{i j j}^{P}\right] \psi(P) .
\end{aligned}
$$

By adding (11.5) multiplied by a free parameter $t$, one finds that the last line of (11.4) is equivalent to

$$
\begin{aligned}
& {\left[P_{i j k} P_{j l m} D_{k l m}^{P}+(3 B+t) P_{i j k} D_{j k z}^{P}\right.} \\
& \left.\quad+\left\{2 B^{2}+A B+t(2 B-A)\right\} D_{i z z}^{P}+\left(B^{2}-t B\right) D_{i j j}^{P}\right] \psi(P) .
\end{aligned}
$$

Here, by choosing $t=-3 B, A=B$, one can delete the second and third terms to obtain

$$
P_{i b c} P_{b d e} D_{c d e}^{P} \psi(P)=\left[P_{i j k} P_{j l m} D_{k l m}^{P}+4 A^{2} D_{i j j}^{P}\right] \psi(P) .
$$

Then, from (11.1), (11.3) and (11.7), we conclude

$$
\left[P_{i j k} P_{j l m} D_{k l m}^{P}+4 A^{2} D_{i j j}^{P}+\lambda_{H}^{N+1} P_{i j j}\right] \psi(P)=0
$$

holds on the subspace (10.17). Finally, we define

$$
\psi_{N}(P)=\psi(P)^{\lambda_{H}^{N} / \lambda_{H}^{N+1}}
$$

Then, $\psi_{N}(P)$ satisfies the constraint equations,

$$
\left[P_{i j k} P_{j l m} D_{k l m}^{P}+4 A^{2} D_{i j j}^{P}+\lambda_{H}^{N} P_{i j j}\right] \psi_{N}(P)=0,
$$

with a cosmological constant,

$$
\lambda=-4 A^{2} .
$$

Two comments are in order. One is that, to obtain a positive cosmological constant, we have to perform analytic continuation of $P_{z z z}=P_{z i i}=A$ to pure imaginary values. On the other hand, the explicit general solutions with a cosmological constant for $N=2,3$ in section 3 and 4 show that $\lambda$ appears without such imaginary numbers. This suggests that there might be another more improved discussion to ignore a cosmological constant than above. The second is that it is totally unclear whether all the solutions to the constraint equations with a cosmological constant can be obtained from those with no cosmological constant by the present method of increasing $N$. What we have shown is merely the reverse; the latter to the former. Therefore, at present, we do not know whether we can totally ignore a cosmological constant in the analysis of the canonical tensor model. We leave this interesting possibility for future study. 


\section{Summary and discussions}

In this paper, we studied exact physical states in the canonical tensor model. Our interest in this paper was to study the canonical tensor model of higher $N$ with and without a cosmological constant, and wherever possible to find the exact general solutions to the constraint equations. After introduction, we presented an outline of the canonical tensor model in section 2. Here we described the constraint algebra and the corresponding equations satisfied by the physical states of the canonical tensor model. Here we also introduced the concept of kinematical and dynamical parts of the states, where the latter reflects the non-linear characteristics of the constraints and would be physically more relevant. We then proceeded to study the canonical tensor model for various $N$. The achievements of this paper can be summarized into the following three categories.

The first is that we have explicitly obtained the physical wave-functions for $N=2,3$, and have studied their features. We considered the cases with and without a cosmological constant, and explicitly wrote down and solved the set of the partial differential equations representing the constraints. While the cases without a cosmological constant were more or less straightforward to solve in a standard method (was indeed previously solved for $N=2$ in [45]), the cases with a cosmological constant set a new challenge. In order to solve them, we solved on a $Z_{2}\left(Z_{3}\right)$-symmetric subspace in the configuration space for $N=2(N=3$, resp. $)$ and then extended the solution over the whole space for $N=2$. Remarkably, the solutions in both the cases with and without a cosmological constant turned out to have the same form when expressed with some functions in which all the effects of the cosmological constant are included. This actually hints that the inclusion of the cosmological constant does not change the structure of the system and tickles our intuition that this can be true even for higher- $N$ tensor models. In order to investigate this issue we went through a different path in the last sections.

Since we did not assume any boundary (or initial) conditions, the solutions contain undetermined functions. However, we were able to study the possible locations of the peaks of the wave-functions, which would be physically interpreted as enhanced configurations. We have found that, for $N=3$ with no cosmological constant, the configurations invariant under an $S O(2)$ symmetry as well as those associated to a Grassmann algebra (this also appears for $N=2$ ) can be enhanced. Moreover, the intersecting points of these two cases have additionally a Lorentz boost symmetry. While the $S O(2)$ and Lorentz boost transformations commute with each other for $N=3$, and thereby do not give rise to any interesting pictures, we expect more non-trivial phenomena to arise in higher- $N$ tensor models.

The second is that we have systematically constructed some series of solutions valid for any $N$ from the perspective of statistical systems on random networks, or random tensor networks. It was noticed that the canonical tensor model shares some resemblances with the statistical systems on random networks, where the Hamiltonian vector flow of the canonical tensor model corresponds to the renormalisation group flow of the statistical system [47]. The "grand-type" partition function of the statistical system (5.1) satisfies the momentum constraints of the tensor model. However, it doesn't satisfy the Hamiltonian constraints. This was cured by integrating the "grand-type" partition function in a scale- 
free manner, which provided a physical wave-function of the canonical tensor model with no cosmological constant. This is a remarkable achievement, as it gives a series of solutions valid for any $N$. An important fact noted in the analysis is that the solutions constructed in this manner are dynamical at least for $N \geq 3$. An interesting connection between the "grand-type" partition function and an extension of Airy function was also noticed by transforming the integration variables.

The above process of constructing physical wave-functions was simplified and generalized. We have constructed more general solutions valid for any $N$ and no cosmological constant in terms of simpler expressions with integration over an $N$-component vector. These solutions are dynamical at least for $N \geq 3$ as above. A similar process of constructing wave-functions for no cosmological constant has been performed in the conjugate (momentum) representation. However, these solutions turned out to be kinematical. Then we also considered integration over matrix and tensor variables, and found a new dynamical solution as well as kinematical solutions. We pointed out that, though kinematical solutions might be of less physical interest than dynamical ones, the former can extend the variations of the latter with continuous parameters.

The third is that we have performed a general analysis of the wave-functions in the momentum representation for the cases with a cosmological constant. We started with the similar assumptions about the forms of wave-functions and solved the constraint equations. Remarkably, the solutions for $N=m$ with a cosmological constant turned out to be obtained by restricting the domains of the solutions for $N=m+1$ with no cosmological constant. Then we have proven a general theorem that the latter can always generate the former by such restriction. This suggests that the effect of the cosmological constant can be absorbed into the dynamics of the tensor model with increased $N$. This is somewhat like in general relativity, where a de Sitter background (maybe arising due to a cosmological constant) can be studied by embedding it in a flat space-time of one higher dimension. This property is also true for more general solutions.

It is now evident that there exist at least some tractable solutions valid for any $N$ in the canonical tensor model, though the quantized constraints on physical states are nonlinear and complicated. It would be an interesting question how further we can proceed: is it possible to write down all the solutions in some tractable manners? On the other hand, from the physical point of view, it would rather be important to obtain a unique physical state which satisfies a physically required initial condition. Then, one can discuss not only the possibilities but the real configurations which become the peaks of the wavefunction. This will inevitably be related with the space-time emergence in the canonical tensor model, and as explicitly shown for $N=3$, there would be symmetry enhancement, which would be related with why the universe is a homogenous space-time with various gauge symmetries. This enhancement would also suggest possible connections between the canonical tensor model and the group field theories [20-35], in which Lie groups are embedded as input structures. Then, what is the initial condition? This question would also be tightly related to what is time in the canonical tensor model, which contains no preferred time variable in its basic formulation. Finally, the discussions on a cosmological constant in this paper strongly suggests that it can totally be ignored. This would be a 
really interesting possibility in view of the so-called cosmological constant problem, and we hope to complete the discussions in future study.

\section{Acknowledgments}

GN would like to thanks NS, Shinya Aoki and others at the Yukawa Institute for Theoretical Physics (YITP) in Kyoto University for providing the wonderful hospitality where a part of the work was done, and is grateful to them for providing the generous financial support. YS is very grateful to YITP, where a part of this work was done, for their warm hospitality, and would like to acknowledge financial support from YITP: the bilateral international exchange program (BIEP), and the long-term international exchange program for young researchers.

Open Access. This article is distributed under the terms of the Creative Commons Attribution License (CC-BY 4.0), which permits any use, distribution and reproduction in any medium, provided the original author(s) and source are credited.

\section{References}

[1] J. Ambjørn, B. Durhuus and T. Jonsson, Three-dimensional simplicial quantum gravity and generalized matrix models, Mod. Phys. Lett. A 6 (1991) 1133 [INSPIRE].

[2] N. Sasakura, Tensor model for gravity and orientability of manifold, Mod. Phys. Lett. A 6 (1991) 2613 [inSPIRE].

[3] N. Godfrey and M. Gross, Simplicial quantum gravity in more than two-dimensions, Phys. Rev. D 43 (1991) 1749 [inSPIRE].

[4] P. Di Francesco, P.H. Ginsparg and J. Zinn-Justin, $2 D$ gravity and random matrices, Phys. Rept. 254 (1995) 1 [hep-th/9306153] [INSPIRE].

[5] R. De Pietri and C. Petronio, Feynman diagrams of generalized matrix models and the associated manifolds in dimension 4, J. Math. Phys. 41 (2000) 6671 [gr-qc/0004045] [INSPIRE].

[6] R. Gurau, Colored group field theory, Commun. Math. Phys. 304 (2011) 69 [arXiv: 0907.2582] [INSPIRE].

[7] R. Gurau and J.P. Ryan, Colored tensor models - A review, SIGMA 8 (2012) 020 [arXiv: 1109.4812] [INSPIRE].

[8] V. Bonzom, R. Gurau, A. Riello and V. Rivasseau, Critical behavior of colored tensor models in the large-N limit, Nucl. Phys. B 853 (2011) 174 [arXiv:1105.3122] [INSPIRE].

[9] R. Gurau and J.P. Ryan, Melons are branched polymers, Annales Henri Poincaré 15 (2014) 2085 [arXiv: 1302.4386] [INSPIRE].

[10] M. Raasakka and A. Tanasa, Next-to-leading order in the large- $N$ expansion of the multi-orientable random tensor model, arXiv:1310.3132 [INSPIRE].

[11] S. Dartois, R. Gurau and V. Rivasseau, Double scaling in tensor models with a quartic interaction, JHEP 09 (2013) 088 [arXiv:1307.5281] [INSPIRE].

[12] W. Kamiński, D. Oriti and J.P. Ryan, Towards a double-scaling limit for tensor models: probing sub-dominant orders, New J. Phys. 16 (2014) 063048 [arXiv:1304.6934] [InSPIRE]. 
[13] V. Bonzom and F. Combes, Tensor models from the viewpoint of matrix models: the case of loop models on random surfaces, arXiv:1304.4152 [INSPIRE].

[14] R. Gurau, The $1 / N$ expansion of tensor models beyond perturbation theory, Commun. Math. Phys. 330 (2014) 973 [arXiv:1304.2666] [INSPIRE].

[15] S. Dartois, V. Rivasseau and A. Tanasa, The $1 / N$ expansion of multi-orientable random tensor models, Annales Henri Poincaré 15 (2014) 965 [arXiv:1301.1535] [INSPIRE].

[16] V. Bonzom, New 1/N expansions in random tensor models, JHEP 06 (2013) 062 [arXiv:1211.1657] [INSPIRE].

[17] A. Tanasa, Multi-orientable group field theory, J. Phys. A 45 (2012) 165401 [arXiv: 1109.0694] [INSPIRE].

[18] S. Dartois, A Givental-like formula and bilinear identities for tensor models, arXiv:1409.5621 [INSPIRE].

[19] V.A. Nguyen, S. Dartois and B. Eynard, An analysis of the intermediate field theory of $T^{4}$ tensor model, arXiv:1409.5751 [INSPIRE].

[20] S. Carrozza, Discrete renormalization group for $\mathrm{SU}(2)$ tensorial group field theory, arXiv:1407.4615 [INSPIRE].

[21] V. Rivasseau, The tensor track, III, Fortsch. Phys. 62 (2014) 81 [arXiv:1311.1461] [INSPIRE].

[22] S. Carrozza, Tensorial methods and renormalization in group field theories, arXiv:1310.3736 [INSPIRE].

[23] J. Ben Geloun, On the finite amplitudes for open graphs in Abelian dynamical colored Boulatov-Ooguri models, J. Phys. A 46 (2013) 402002 [arXiv:1307.8299] [InSPIRE].

[24] J. Ben Geloun, Renormalizable models in rank $d \geq 2$ tensorial group field theory, Commun. Math. Phys. 332 (2014) 117 [arXiv:1306.1201] [INSPIRE].

[25] D. Ousmane Samary, $\beta$-functions ofU $(1)^{d}$ gauge invariant just renormalizable tensor models, Phys. Rev. D 88 (2013) 105003 [arXiv:1303.7256] [INSPIRE].

[26] S. Carrozza, D. Oriti and V. Rivasseau, Renormalization of a SU(2) tensorial group field theory in three dimensions, Commun. Math. Phys. 330 (2014) 581 [arXiv:1303.6772] [INSPIRE].

[27] S. Carrozza, D. Oriti and V. Rivasseau, Renormalization of tensorial group field theories: abelian U(1) models in four dimensions, Commun. Math. Phys. 327 (2014) 603 [arXiv:1207.6734] [INSPIRE].

[28] J. Ben Geloun and E.R. Livine, Some classes of renormalizable tensor models, J. Math. Phys. 54 (2013) 082303 [arXiv:1207.0416] [InSPIRE].

[29] J. Ben Geloun and D.O. Samary, 3D tensor field theory: renormalization and one-loop $\beta$-functions, Annales Henri Poincaré 14 (2013) 1599 [arXiv: 1201.0176] [INSPIRE].

[30] J. Ben Geloun and V. Rivasseau, A renormalizable 4-dimensional tensor field theory, Commun. Math. Phys. 318 (2013) 69 [arXiv:1111.4997] [INSPIRE].

[31] D.V. Boulatov, A Model of three-dimensional lattice gravity, Mod. Phys. Lett. A 7 (1992) 1629 [hep-th/9202074] [INSPIRE].

[32] H. Ooguri, Topological lattice models in four-dimensions, Mod. Phys. Lett. A 7 (1992) 2799 [hep-th/9205090] [INSPIRE]. 
[33] R. De Pietri, L. Freidel, K. Krasnov and C. Rovelli, Barrett-Crane model from a Boulatov-Ooguri field theory over a homogeneous space, Nucl. Phys. B 574 (2000) 785 [hep-th/9907154] [INSPIRE].

[34] L. Freidel, Group field theory: an overview, Int. J. Theor. Phys. 44 (2005) 1769 [hep-th/0505016] [INSPIRE].

[35] D. Oriti, The microscopic dynamics of quantum space as a group field theory, arXiv:1110.5606 [INSPIRE].

[36] J. Ambjørn, J. Jurkiewicz and R. Loll, Emergence of a $4 D$ world from causal quantum gravity, Phys. Rev. Lett. 93 (2004) 131301 [hep-th/0404156] [INSPIRE].

[37] S. Horata, H.S. Egawa, N. Tsuda and T. Yukawa, Phase structure of four-dimensional simplicial quantum gravity with a U(1) gauge field, Prog. Theor. Phys. 106 (2001) 1037 [hep-lat/0004021] [INSPIRE].

[38] J. Ambjørn, K.N. Anagnostopoulos and J. Jurkiewicz, Abelian gauge fields coupled to simplicial quantum gravity, JHEP 08 (1999) 016 [hep-lat/9907027] [INSPIRE].

[39] N. Sasakura, Canonical tensor models with local time, Int. J. Mod. Phys. A 27 (2012) 1250020 [arXiv:1111.2790] [InSPIRE].

[40] N. Sasakura, Uniqueness of canonical tensor model with local time, Int. J. Mod. Phys. A 27 (2012) 1250096 [arXiv: 1203.0421] [INSPIRE].

[41] N. Sasakura, A canonical rank-three tensor model with a scaling constraint, Int. J. Mod. Phys. A 28 (2013) 1350030 [arXiv:1302.1656] [InSPIRE].

[42] D. Oriti, Group field theory as the 2nd quantization of loop quantum gravity, arXiv: 1310.7786 [INSPIRE].

[43] R.L. Arnowitt, S. Deser and C.W. Misner, Canonical variables for general relativity, Phys. Rev. 117 (1960) 1595 [INSPIRE].

[44] R.L. Arnowitt, S. Deser and C.W. Misner, The dynamics of general relativity, Gen. Rel. Grav. 40 (2008) 1997 [gr-qc/0405109] [inSPIRE].

[45] N. Sasakura, Quantum canonical tensor model and an exact wave function, Int. J. Mod. Phys. A 28 (2013) 1350111 [arXiv:1305.6389] [inSPIRE].

[46] N. Sasakura and Y. Sato, Interpreting canonical tensor model in minisuperspace, Phys. Lett. B 732 (2014) 32 [arXiv:1401.2062] [INSPIRE].

[47] N. Sasakura and Y. Sato, Ising model on random networks and the canonical tensor model, PTEP 2014 (2014) 053B03 [arXiv:1401.7806] [INSPIRE].

[48] N. Sasakura and Y. Sato, Exact free energies of statistical systems on random networks, SIGMA 10 (2014) 087 [arXiv: 1402.0740] [INSPIRE].

[49] S.N. Dorogovtsev, A.V. Goltsev and J.F.F. Mendes, Critical phenomena in complex networks, Rev. Mod. Phys. 80 (2008) 1275 [InSPIRE].

[50] B.S. DeWitt, Quantum theory of gravity. 1. The canonical theory, Phys. Rev. 160 (1967) 1113 [INSPIRE].

[51] N. Sasakura, Tensor models and 3-ary algebras, J. Math. Phys. 52 (2011) 103510 [arXiv: 1104.1463] [INSPIRE]. 